\title{
On the Higher-Order Inhomogeneous Heisenberg Supermagnetic Models
}

\author{
Rong $\operatorname{Han}^{1}$ (D) Haichao Sun ${ }^{1} \cdot$ Nana Jiang ${ }^{1} \cdot$ Zhaowen Yan $^{1}$
}

Received: 15 November 2020 / Accepted: 31 May 2021 / Published online: 18 August 2021

(c) The Author(s) 2021

\begin{abstract}
This paper is concerned with the construction of the fifth-order inhomogeneous Heisenberg supermagnetic models. Moreover, the Lax representations of the models are presented. By means of the gauge transformation, we establish their gauge equivalent equations with different quadratic constraints, i.e., the super and fermionic fifth-order inhomogeneous nonlinear Schrödinger equations, respectively. In addition, we investigate their Lax representations and Bäcklund transformations from which the solutions of the super integrable systems have been discussed.
\end{abstract}

Keywords Nonlinear Schrödinger equation · Gauge transformation · Heisenberg supermagnetic model

Mathematics Subject Classification $17 \mathrm{~B} 80 \cdot 35 \mathrm{Q} 55 \cdot 37 \mathrm{~K} 10$

\section{Introduction}

The Heisenberg ferromagnet (HF) model $[1,2]$

$$
\mathbf{S}_{t}=\mathbf{S} \times \mathbf{S}_{x x}, \mathbf{S}=\left(S_{1}, S_{2}, S_{3}\right), \mathbf{S} \cdot \mathbf{S}=1 .
$$

is an important integrable system, which describes the movement of the magnetization vector of the isotropic ferromagnets. The HF model has been well developed and it is geometrical and gauge equivalent to the nonlinear Schrödinger equation (NLSE) [3, 4]. There have been extensive study and application of the HF models and the inhomogeneous integrable equations [5, 6], such as deformed HF model [7, 8], extended high-order HF model [9-11], inhomogeneous deformed HF model [3, 12], the multidimensional HF model [13, 14], the multi-component extended HF model [15], and integrable counterparts of the Heisenberg soliton hierarchy [16].

Zhaowen Yan

yanzw@imu.edu.cn

1 School of Mathematical Sciences, Inner Mongolia University, Hohhot 010021, China 
Qiao et al. investigated the involutive solutions of the higher-order HF model in terms of the spectral problem nonlinearization approach [17]. Then it is showed the constrained HF hierarchy possess the same $r$-matrix with the constrained Harry-Dym system [18].

Supersymmetry plays a significant role between theoretical physics and mathematics [19-21]. Thus integrable supersymmetric systems have attracted considerable attention in the mathematical as well as physical points of view. A number of famous integrable systems have been generalized to their supersymmetric version, such as the Korteweg-de Vries (KdV) equation [22, 23], the Kadomtsev-Petviashvili (KP) equation [24], the HF model [25-27] and the inhomogeneous nonlinear Schrödinger type equation [28]. Ma et al. [29] investigate the applications to super integrable systems by means of the supertrace identity on Lie super algebras.

The Heisenberg supermagnet (HS) model can be regarded as the supersymmetric extension of the HF model $[11,25]$. The HS models and their corresponding gauge equivalence were first developed by Makhankov et al. [25]. Furthermore, the higher order and inhomogeneous HS models have been discussed and their integrable structure and properties have been also derived. Meanwhile, the authors [11,30] constructed the third-order, fourth-order and fifth-order generalized HS models from which their gauge equivalent equations have been presented. Moreover, Yan et al. developed the inhomogeneous thirdorder and fourth-order generalized HS models [26, 27], respectively. The corresponding gauge equivalent equations are super and fermionic inhomogeneous NLSEs. Therefore, our purpose of this paper is to develop inhomogeneous deformations of fifth-order HS model and analyze their structure and integral properties. Furthermore, we shall derive the Bäcklund transformations of the super nonlinear evolution equation.

The organization of this paper is as follows. In the second section, the HS model is briefly reviewed and its integrable properties are recalled. In the third section, we construct the inhomogeneous fifth-order HS model. Then the gauge equivalent equations with two quadratic constraints and Bäcklund transformations are derived. In the fourth section, we dedicate to a summary and discussion.

\section{Heisenberg Supermagnet Model}

Let us start with a short summary of the HS model that will be useful in what follows. For a more detailed description, we refer the reader to [25].

The HS model is described by

$$
i S_{t}=\left[S, S_{x x}\right],
$$

where $S$ is a superspin function which can be given by

$$
\begin{aligned}
S & =2 \sum_{a=1}^{4} S_{a} T_{a}+2 \sum_{a=5}^{8} C_{a} T_{a}, \\
& =\left(\begin{array}{ccc}
S_{3}+S_{4} & S_{1}-i S_{2} & C_{5}-i C_{6} \\
S_{1}+i S_{2} & -S_{3}+S_{4} & C_{7}-i C_{8} \\
C_{5}+i C_{6} & C_{7}+i C_{8} & 2 S_{4}
\end{array}\right),
\end{aligned}
$$


where $S_{1}, \ldots, S_{4}$ are the bosonic variables and $C_{5}, \ldots, C_{8}$ are the fermionic variables. $T_{1}, \ldots, T_{4}$ are bosonic generators of the superalgebra $\operatorname{su}(2 / 1)$ and $T_{5}, \ldots, T_{8}$ are fermionic generators of the superalgebra $s u(2 / 1)$.

The gauge equivalence plays an important role in the integral systems. The fact is that the gauge equivalence exists only for integrable systems possess Lax representations. Understanding the properties of the gauge equivalent counterpart helps us know more about the integrable systems. Under the following two constraints, Makhankov and Pashaev showed the HS model is gauge equivalent to supersymmetric NLSE and Grassman odd NLSE, respectively

(i) $S^{2}=S$ for $S \in S U(2 / 1) / S(L(1 / 1) \times U(1))$

$$
\begin{aligned}
& i \phi_{t}+\phi_{x x}+2(\phi \bar{\phi}+\theta \bar{\theta}) \phi=0, \\
& i \theta_{t}+\theta_{x x}+2 \phi \bar{\phi} \theta=0 .
\end{aligned}
$$

(ii) $S^{2}=3 S-2 I$ for $S \in S U(2 / 1) / S(U(2) \times U(1))$

$$
\begin{aligned}
& i \theta_{1 t}+\theta_{1 x x}+2 \bar{\theta}_{2} \theta_{2} \theta_{1}=0, \\
& i \theta_{2 t}+\theta_{2 x x}+2 \bar{\theta}_{1} \theta_{1} \theta_{2}=0,
\end{aligned}
$$

where $\phi(x, t)$ is a bosonic components and $\theta, \theta_{1}, \theta_{2}$ are the fermionic ones.

\section{Fifth-Order Inhomogeneous Heisenberg Supermagnet Model}

\subsection{Constraints (i) $S^{2}=S$}

Under constraint (i) $S^{2}=S$, let us consider the fifth-order inhomogeneous HS model

$$
i S_{t}=f\left[S, S_{x x}\right]+f_{x}\left[S, S_{x}\right]+i h S_{x}+i \varepsilon S_{x x x x x}+i \varepsilon D\left(S_{x}, S_{x x}, S_{x x x}, S_{x x x x}\right),
$$

where $f$ and $h$ are the functions of $x$ and $t, \varepsilon$ is a parameter. Making use of the constraint (i) $S^{2}=S$, we note that generalized function $D\left(S_{x}, S_{x x}, S_{x x x}, S_{x x x x}\right)$ satisfies

$$
S D+D S=D .
$$

With the constraint (i), it's easy to prove $S_{t}$ and $\left[S, S_{x x}\right]$ satisfies $S S_{t} S=0$ and $S\left[S, S_{x x}\right] S=0$.

The Lax representation of the fifth-order HS model contains no higher than the fourth-order derivatives with respect to $x$.

We now introduce the Lax representation of the HS model (6),

$$
\varphi_{x}=F \varphi, \varphi_{t}=G \varphi,
$$

where $\varphi=\left(\varphi_{1}, \varphi_{2}, \varphi_{3}\right)^{\perp}, \varphi_{j}, j=1,2$ and $\varphi_{3}$ are the bosonic and fermionic functions, respectively, and $\mathrm{F}, \mathrm{G}$ can be presented as 


$$
\begin{aligned}
F= & -i \lambda S, \\
G= & -\lambda f\left[S_{x}, S\right]-\left(i \lambda h+i \lambda^{2} f\right) S+\varepsilon\left(-i \lambda^{5} S+\lambda^{4}\left[S, S_{x}\right]\right) \\
& +\varepsilon \sum_{i=1}^{n} \lambda^{i} Q_{i}\left(S, S_{x}, S_{x x}, S_{x x x}, S_{x x x x}\right),
\end{aligned}
$$

where $\lambda$ is a spectral parameter.

The Lax pair satisfies the zero-curvature equation

$$
F_{t}-G_{x}+[F, G]=0 .
$$

By substituting (9) into (10) and taking advantage of the condition (7), we have

$$
\begin{aligned}
D= & -5\left(S_{x x x x} S_{x}+S_{x} S_{x x x x}\right)-10\left(S_{x x x} S_{x x}+S_{x x} S_{x x x}-S S_{x x x x} S_{x}-S S_{x} S_{x x x x}\right) \\
& +15\left(S_{x} S_{x} S_{x x x}+S_{x x x} S_{x} S_{x}\right)+20\left(S_{x x} S_{x} S_{x x}+S_{x} S_{x x x} S_{x}+S S_{x x x} S_{x x}\right. \\
& \left.+S S_{x x} S_{x x x}\right)+25\left(S_{x} S_{x x} S_{x x}+S_{x x} S_{x x} S_{x}\right)+70\left(S_{x} S_{x} S_{x} S_{x} S_{x}+S S_{x x} S_{x} S_{x} S_{x}\right. \\
& \left.+S S_{x} S_{x x} S_{x} S_{x}+S S_{x} S_{x} S_{x x} S_{x}+S S_{x} S_{x} S_{x} S_{x x}\right)-35\left(S_{x x} S_{x} S_{x} S_{x}+S_{x} S_{x x} S_{x} S_{x}\right. \\
& \left.+S_{x} S_{x} S_{x x} S_{x}+S_{x} S_{x} S_{x} S_{x x}\right) \\
Q_{1}= & i\left(-S_{x x x x}+5 S_{x x x} S_{x}+5 S_{x x} S_{x x}+5 S_{x} S_{x x x}-10 S S_{x x x} S_{x}-10 S S_{x x} S_{x x}\right. \\
& -10 S S_{x} S_{x x x}-15 S_{x x} S_{x} S_{x}-10 S_{x} S_{x x} S_{x}-5 S_{x} S_{x} S_{x x}+35 S_{x} S_{x} S_{x} S_{x} \\
& \left.-70 S S_{x} S_{x} S_{x} S_{x}\right) \\
Q_{2}= & -\left[S, S_{x x x}\right]+\left[S_{x}, S_{x x}\right]+10 S_{x} S_{x} S_{x}-20 S S_{x} S_{x} S_{x} \\
Q_{3}= & i\left(S_{x x}-3 S_{x} S_{x}+6 S S_{x} S_{x}\right) \\
Q_{4}= & Q_{5}=\cdots=Q_{n}=0 \\
\lambda_{t}= & \lambda^{2} f_{x}+\lambda h_{x} .
\end{aligned}
$$

From the Eq. (10) and contrasting coefficients of the power of $\lambda$, we derive the fifthorder inhomogeneous HS model

$$
\begin{aligned}
i S_{t}= & f\left[S, S_{x x}\right]+f_{x}\left[S, S_{x}\right]+i h S_{x}+i \varepsilon\left[S_{x x x x x}-5\left(S_{x x x} S_{x}+S_{x x} S_{x x}+S_{x} S_{x x x}\right.\right. \\
& \left.-S_{x} S_{x} S_{x x}\right)_{x}+10\left(S S_{x x x} S_{x}+S S_{x x} S_{x x}+S S_{x} S_{x x x}+S_{x} S_{x x} S_{x}\right)_{x} \\
& \left.+15\left(S_{x x} S_{x} S_{x}\right)_{x}+35\left(2 S S_{x} S_{x} S_{x} S_{x}-S_{x} S_{x} S_{x} S_{x}\right)_{x}\right] .
\end{aligned}
$$

The corresponding $F$ and $G$ are given by

$$
\begin{aligned}
F= & -i \lambda S, \\
G= & -\lambda f\left[S_{x}, S\right]-\left(i \lambda h+i \lambda^{2} f\right) S+\varepsilon\left[-i \lambda^{5} S+\lambda^{4}\left[S, S_{x}\right]\right. \\
& +i \lambda^{3}\left(S_{x x}-3 S_{x} S_{x}+6 S S_{x} S_{x}\right) \\
& +\lambda^{2}\left(-\left[S, S_{x x x}\right]+\left[S_{x}, S_{x x}\right]+10 S_{x} S_{x} S_{x}-20 S S_{x} S_{x} S_{x}\right)+i \lambda\left(-S_{x x x x}\right. \\
& +5 S_{x x x} S_{x} \\
& +5 S_{x x} S_{x x}+5 S_{x} S_{x x x}-10 S S_{x x x} S_{x}-10 S S_{x x} S_{x x}-10 S S_{x} S_{x x x}-15 S_{x x} S_{x} S_{x} \\
& \left.\left.-10 S_{x} S_{x x} S_{x}-5 S_{x} S_{x} S_{x x}+35 S_{x} S_{x} S_{x} S_{x}-70 S S_{x} S_{x} S_{x} S_{x}\right)\right],
\end{aligned}
$$


where $\lambda$ is a spectral parameter.

In order to derive the gauge equivalent equation of (12), one takes

$$
S(x, t)=g^{-1}(x, t) \sum g(x, t),
$$

where $g(x, t) \in S U(2 / 1)$.

According to condition

$$
J_{1}=g_{x} g^{-1}, \quad J_{0}=g_{t} g^{-1},
$$

Equation (15) satisfies

$$
\partial_{t} J_{1}-\partial_{x} J_{0}+\left[J_{1}, J_{0}\right]=0 .
$$

The orthogonal direct sum decomposition of the super algebra $s u(2 / 1)$ is as follows

$$
\mathrm{L}=\mathrm{L}^{(0)} \oplus \mathrm{L}^{(1)},
$$

where $\left[\mathrm{L}^{(0)}, \mathrm{L}^{(0)}\right] \subset \mathrm{L}^{(0)},\left[\mathrm{L}^{(0)}, \mathrm{L}^{(1)}\right] \subset \mathrm{L}^{(1)},\left[\mathrm{L}^{(1)}, \mathrm{L}^{(1)}\right]_{+} \subset \mathrm{L}^{(0)}$. The commutation and anticommutator relations are given by $[X, Y]=X Y-Y X,[X, Y]_{+}=X Y+Y X . L^{(0)}$ is an algebra constructed in terms of the stationary subgroup $H$.

Suppose

$$
J_{1}=i\left(\begin{array}{ccc}
0 & \phi & \theta \\
\bar{\phi} & 0 & 0 \\
\bar{\theta} & 0 & 0
\end{array}\right) \in \mathrm{L}^{(1)} \text { for } S \in S U(2 / 1) / S(L(1 / 1) \times U(1)),
$$

where $\phi(x, t)$ is bosonic filed and $\theta(x, t)$ is fermionic one.

Based on (14), (15) and (18), we have

$$
\begin{aligned}
S_{t}= & g^{-1}(x, t)\left[\Sigma, J_{0}\right] g(x, t), \\
S_{x}= & g^{-1}(x, t)\left[\Sigma, J_{1}\right] g(x, t), \\
S_{x x}= & g^{-1}(x, t)\left(\left[\left[\Sigma, J_{1}\right], J_{1}\right]+\left[\Sigma, J_{1 x}\right]\right) g(x, t), \\
S_{x x x}= & g^{-1}(x, t)\left(\left[\Sigma, J_{1 x x}\right]+\left[\left[\left[\Sigma, J_{1}\right], J_{1}\right], J_{1}\right]\right. \\
& \left.+2\left[\left[\Sigma, J_{1 x}\right], J_{1}\right]+\left[\left[\Sigma, J_{1}\right], J_{1 x}\right]\right) g(x, t), \\
S_{x x x x}= & g^{-1}(x, t)\left(3\left[\left[\left[\Sigma, J_{1 x}\right], J_{1}\right], J_{1}\right]+3\left[\left[\Sigma, J_{1 x x}\right], J_{1}\right]\right. \\
& \left.+\left[\left[\left[\left[\Sigma, J_{1}\right], J_{1}\right], J_{1}\right], J_{1}\right]\right) \\
& +2\left[\left[\left[\Sigma, J_{1}\right], J_{1 x}\right], J_{1}\right]+3\left[\left[\Sigma, J_{1 x}\right], J_{1 x}\right]+\left[\Sigma, J_{1 x x x}\right] \\
& \left.+\left[\left[\left[\Sigma, J_{1}\right], J_{1}\right], J_{1 x}\right]+\left[\left[\Sigma, J_{1}\right], J_{1 x x}\right]\right) g(x, t), \\
S_{x x x x x}= & g^{-1}(x, t)\left(4\left[\left[\left[\left[\Sigma, J_{1 x}\right], J_{1}\right], J_{1}\right], J_{1}\right]+6\left[\left[\left[\Sigma, J_{1 x x}\right], J_{1}\right], J_{1}\right]\right. \\
& +\left[\left[\left[\left[\left[\Sigma, J_{1}\right], J_{1}\right], J_{1}\right], J_{1}\right], J_{1}\right]+3\left[\left[\left[\left[\Sigma, J_{1}\right], J_{1 x},\right], J_{1}\right], J_{1}\right] \\
& +8\left[\left[\left[\Sigma, J_{1 x}\right], J_{1 x}\right], J_{1}\right] \\
& +4\left[\left[\Sigma, J_{1 x x x}\right], J_{1}\right]+2\left[\left[\left[\left[\Sigma, J_{1}\right], J_{1}\right], J_{1 x}\right], J_{1}\right]+3\left[\left[\left[\Sigma, J_{1}\right], J_{1 x x}\right], J_{1}\right] \\
& +4\left[\left[\left[\Sigma, J_{1 x}\right], J_{1}\right], J_{1 x}\right]+6\left[\left[\Sigma, J_{1 x x}\right], J_{1 x}\right]+\left[\left[\left[\left[\Sigma, J_{1}\right], J_{1}\right], J_{1}\right], J_{1 x}\right] \\
& +3\left[\left[\left[\Sigma, J_{1}\right], J_{1 x}\right], J_{1 x}\right]+4\left[\left[\Sigma, J_{1 x}\right], J_{1 x x}\right]+\left[\Sigma, J_{1 x x x x}\right] \\
& \left.+\left[\left[\left[\Sigma, J_{1}\right], J_{1}\right], J_{1 x x}\right]+\left[\left[\Sigma, J_{1}\right], J_{1 x x x}\right]\right) g(x, t) .
\end{aligned}
$$


Substituting (14) and (19) into (12), we obtain

$$
\begin{aligned}
= & -i f\left[\Sigma,\left[\left[\Sigma, J_{1}\right], J_{1}\right]+\left[\Sigma, J_{1 x}\right]\right]-i f_{x}\left[\Sigma,\left[\Sigma, J_{1}\right]\right]+h\left[\Sigma, J_{1}\right] \\
& +\varepsilon\left[4\left[\left[\left[\left[\Sigma, J_{1 x}\right], J_{1}\right], J_{1}\right], J_{1}\right]+6\left[\left[\left[\Sigma, J_{1 x x}\right], J_{1}\right], J_{1}\right]\right. \\
& +\left[\left[\left[\left[\left[\Sigma, J_{1}\right], J_{1}\right], J_{1}\right], J_{1}\right], J_{1}\right] \\
& +3\left[\left[\left[\left[\Sigma, J_{1}\right], J_{1 x},\right], J_{1}\right], J_{1}\right]+8\left[\left[\left[\Sigma, J_{1 x}\right], J_{1 x}\right], J_{1}\right]+4\left[\left[\Sigma, J_{1 x x x}\right], J_{1}\right] \\
& +2\left[\left[\left[\left[\Sigma, J_{1}\right], J_{1}\right], J_{1 x}\right], J_{1}\right]+3\left[\left[\left[\Sigma, J_{1}\right], J_{1 x x}\right], J_{1}\right] \\
& +4\left[\left[\left[\Sigma, J_{1 x}\right], J_{1}\right], J_{1 x}\right] \\
& +6\left[\left[\Sigma, J_{1 x x}\right], J_{1 x}\right]+\left[\left[\left[\left[\Sigma, J_{1}\right], J_{1}\right], J_{1}\right], J_{1 x}\right]+3\left[\left[\left[\Sigma, J_{1}\right], J_{1 x}\right], J_{1 x}\right] \\
& +4\left[\left[\Sigma, J_{1 x}\right], J_{1 x x}\right]+\left[\Sigma, J_{1 x x x}\right]+\left[\left[\left[\Sigma, J_{1}\right], J_{1}\right], J_{1 x x}\right] \\
& +\left[\left[\Sigma, J_{1}\right], J_{1 x x x}\right] \\
& +5\left[\left(\left[\Sigma, J_{1 x x}\right]+\left[\left[\left[\Sigma, J_{1}\right], J_{1}\right], J_{1}\right]+2\left[\left[\Sigma, J_{1 x}\right], J_{1}\right]\right.\right. \\
& \left.+\left[\left[\Sigma, J_{1}\right], J_{1 x}\right]\right)\left[\Sigma, J_{1}\right] \\
& +\left(\left[\left[\Sigma, J_{1}\right], J_{1}\right]+\left[\Sigma, J_{1 x}\right]\right)\left(\left[\left[\Sigma, J_{1}\right], J_{1}\right]+\left[\Sigma, J_{1 x}\right]\right)+\left[\Sigma, J_{1}\right]\left(\left[\Sigma, J_{1 x x}\right]\right. \\
& \left.+\left[\left[\left[\Sigma, J_{1}\right], J_{1}\right], J_{1}\right]+2\left[\left[\Sigma, J_{1 x}\right], J_{1}\right]+\left[\left[\Sigma, J_{1}\right], J_{1 x}\right]\right)-\left[\Sigma, J_{1}\right]\left[\Sigma, J_{1}\right] \\
& \left.\times\left(\left[\left[\Sigma, J_{1}\right], J_{1}\right]+\left[\Sigma, J_{1 x}\right]\right)\right]_{x}+10\left(\Sigma \left(\left[\Sigma, J_{1 x x}\right]+\left[\left[\left[\Sigma, J_{1}\right], J_{1}\right], J_{1}\right]\right.\right. \\
& \left.+2\left[\left[\Sigma, J_{1 x}\right], J_{1}\right]+\left[\left[\Sigma, J_{1}\right], J_{1 x}\right]\right)\left[\Sigma, J_{1}\right]+\Sigma\left(\left[\left[\Sigma, J_{1}\right], J_{1}\right]+\left[\Sigma, J_{1 x}\right]\right) \\
& \times\left(\left[\left[\Sigma, J_{1}\right], J_{1}\right]+\left[\Sigma, J_{1 x}\right]\right)+\Sigma\left[\Sigma, J_{1}\right]\left(\left[\Sigma, J_{1 x x}\right]+\left[\left[\left[\Sigma, J_{1}\right], J_{1}\right], J_{1}\right]\right. \\
& \left.+2\left[\left[\Sigma, J_{1 x}\right], J_{1}\right]+\left[\left[\Sigma, J_{1}\right], J_{1 x}\right]\right)+\left[\Sigma, J_{1}\right]\left(\left[\left[\Sigma, J_{1}\right], J_{1}\right]\right. \\
& \left.\left.+\left[\Sigma, J_{1 x}\right]\right)\left[\Sigma, J_{1}\right]\right)_{x} \\
& +15\left(\left(\left[\left[\Sigma, J_{1}\right], J_{1}\right]+\left[\Sigma, J_{1 x}\right]\right)\left[\Sigma, J_{1}\right]\left[\Sigma, J_{1}\right]\right)_{x} \\
& +35\left(2 \Sigma\left[\Sigma, J_{1}\right]\left[\Sigma, J_{1}\right]\left[\Sigma, J_{1}\right]\right. \\
& \left.\left.\times\left[\Sigma, J_{1}\right]-\left[\Sigma, J_{1}\right]\left[\Sigma, J_{1}\right]\left[\Sigma, J_{1}\right]\left[\Sigma, J_{1}\right]\right)_{x}\right],
\end{aligned}
$$

where $\Sigma=\operatorname{diag}(0,1,1)$.

Combining Eq. (20) with $\left[\Sigma, J_{0}^{(0)}\right]=0$, we obtain

$$
J_{0}^{(1)}=\left(\begin{array}{ccc}
0 & \left(J_{0}^{(1)}\right)_{12} & \left(J_{0}^{(1)}\right)_{13} \\
\left(J_{0}^{(1)}\right)_{21} & 0 & 0 \\
\left(J_{0}^{(1)}\right)_{31} & 0 & 0
\end{array}\right),
$$

where 


$$
\begin{aligned}
\left(J_{0}^{(1)}\right)_{12}= & -(f \phi)_{x}+i h \phi+\varepsilon\left[6 i(\phi \bar{\phi} \phi \bar{\phi} \phi+2 \theta \bar{\theta} \phi \bar{\phi} \phi)+i \phi_{x x x x}+2 i\left[\left(\phi \bar{\phi}_{x}\right)_{x} \phi\right.\right. \\
& \left.\left.+\left(\phi_{x} \bar{\phi}\right)_{x} \phi+3\left(\phi_{x} \phi\right)_{x} \bar{\phi}+2\left(\theta_{x} \phi\right)_{x} \bar{\theta}+\left(\theta \phi_{x}\right)_{x} \bar{\theta}+\theta\left(\bar{\theta} \phi_{x}\right)_{x}+\left(\theta \bar{\theta}_{x}\right)_{x} \phi\right]\right], \\
\left(J_{0}^{(1)}\right)_{13}= & -(f \theta)_{x}+i h \theta+\varepsilon\left[6 i \phi \bar{\phi} \phi \bar{\phi} \theta+i \theta_{x x x x}+2 i\left[2\left(\phi_{x} \theta\right)_{x} \bar{\phi}+\left(\phi \bar{\phi}_{x}\right)_{x} \theta\right.\right. \\
& \left.\left.+\left(\bar{\phi} \theta_{x}\right)_{x} \phi+\left(\phi \theta_{x}\right)_{x} \bar{\phi}\right]\right], \\
\left(J_{0}^{(1)}\right)_{21}= & (f \bar{\phi})_{x}+i h \bar{\phi}+\varepsilon\left[6 i(\bar{\phi} \phi \bar{\phi} \phi \bar{\phi}+2 \bar{\phi} \phi \bar{\phi} \theta \bar{\theta})+i \bar{\phi}_{x x x x}+2 i\left[\left(\phi_{x} \bar{\phi}\right)_{x} \bar{\phi}\right.\right. \\
& \left.\left.+\left(\phi \bar{\phi}_{x}\right)_{x} \bar{\phi}+3\left(\bar{\phi}_{x} \bar{\phi}\right)_{x} \phi+2 \theta\left(\bar{\phi} \bar{\theta}_{x}\right)_{x}+\theta\left(\bar{\phi}_{x} \bar{\theta}\right)_{x}+\left(\bar{\phi}_{x} \theta\right)_{x} \bar{\theta}+\left(\theta_{x} \bar{\theta}\right)_{x} \bar{\phi}\right]\right], \\
\left(J_{0}^{(1)}\right)_{31}= & (f \bar{\theta})_{x}+i h \bar{\theta}+\varepsilon\left[6 i \bar{\phi} \phi \bar{\phi} \phi \bar{\theta}+i \bar{\theta}_{x x x x}+2 i\left[2\left(\bar{\phi}_{x} \bar{\theta}\right)_{x} \phi+\left(\bar{\phi} \phi_{x}\right)_{x} \bar{\theta}\right.\right. \\
& \left.\left.+\left(\phi \bar{\theta}_{x}\right)_{x} \bar{\phi}+\left(\bar{\phi} \bar{\theta}_{x}\right)_{x} \phi\right]\right] .
\end{aligned}
$$

According to the Eqs. (16) and (17), we obtain

$$
\left(J_{0}^{(0)}\right)_{x}=\left[J_{1}, J_{0}^{(1)}\right] .
$$

By substituting (18), (21) into (23) and integrating Eq. (23) in reference to respect to the variable $x$, we derive

$$
J_{0}^{(0)}=\left(\begin{array}{ccc}
\left(J_{0}^{(0)}\right)_{11} & 0 & 0 \\
0 & \left(J_{0}^{(0)}\right)_{22} & \left(J_{0}^{(0)}\right)_{23} \\
0 & \left(J_{0}^{(0)}\right)_{32} & \left(J_{0}^{(0)}\right)_{33}
\end{array}\right),
$$

where

$$
\begin{aligned}
\left(J_{0}^{(0)}\right)_{11}= & i f(\phi \bar{\phi}+\theta \bar{\theta})+i \int_{-\infty}^{x} f_{x}(\phi \bar{\phi}+\theta \bar{\theta}) d x^{\prime}+\varepsilon\left[6 \left(\phi_{x} \phi \bar{\phi} \bar{\phi}-\bar{\phi}_{x} \bar{\phi} \phi \phi+\phi_{x} \theta \bar{\phi} \bar{\theta}\right.\right. \\
& \left.+\bar{\phi} \phi_{x x x}-\bar{\phi}_{x x x} \phi-\bar{\phi}_{x} \phi_{x x}+\bar{\phi}_{x x} \phi_{x}+\theta_{x x x} \bar{\theta}+\phi \theta_{x} \bar{\phi} \bar{\theta}-\theta \phi \bar{\phi}_{x} \bar{\theta}-\theta \phi \bar{\phi} \bar{\theta}_{x}\right) \\
& \left.-\theta \bar{\theta}_{x x x}-\theta_{x x} \bar{\theta}_{x}+\theta_{x} \bar{\theta}_{x x}\right], \\
\left(J_{0}^{(0)}\right)_{22}= & -i f \bar{\phi} \phi-i \int_{-\infty}^{x} f_{x} \bar{\phi} \phi d x^{\prime}+\varepsilon\left[6\left(\bar{\phi}_{x} \bar{\phi} \phi \phi-\phi_{x} \phi \bar{\phi} \bar{\phi}\right)+4\left(\phi \theta \bar{\phi}_{x} \bar{\theta}-\phi_{x} \theta \bar{\phi} \bar{\theta}\right)\right. \\
& \left.+2\left(\phi \theta \bar{\phi} \bar{\theta}_{x}-\theta_{x} \phi \bar{\phi} \bar{\theta}\right)+\bar{\phi}_{x x x} \phi-\bar{\phi} \phi_{x x x}-\bar{\phi}_{x x} \phi_{x}+\bar{\phi}_{x} \phi_{x x}\right], \\
\left(J_{0}^{(0)}\right)_{23}= & -i f \bar{\phi} \theta-i \int_{-\infty}^{x} f_{x} \bar{\phi} \theta d x^{\prime}+\varepsilon\left[4\left(\bar{\phi}_{x} \bar{\phi} \phi \theta-\phi \theta_{x} \bar{\phi} \bar{\phi}\right)+2\left(\bar{\phi}_{x} \bar{\phi} \phi \theta-\phi_{x} \theta \bar{\phi} \bar{\phi}\right.\right. \\
& \left.\left.+\theta_{x} \bar{\theta} \bar{\phi} \theta\right)+\bar{\phi}_{x x x} \theta-\bar{\phi} \theta_{x x x}-\bar{\phi}_{x x} \theta_{x}+\bar{\phi}_{x} \theta_{x x}\right], \\
\left(J_{0}^{(0)}\right)_{32}= & -i f \bar{\theta} \phi-i \int_{-\infty}^{x} f_{x} \bar{\theta} \phi d x^{\prime}+\varepsilon\left[4\left(\bar{\phi} \bar{\theta}_{x} \phi \theta-\bar{\theta} \bar{\phi} \phi \theta_{x}\right)+2\left(\bar{\phi}_{x} \bar{\theta} \phi \theta-\bar{\theta} \bar{\phi} \phi_{x} \theta\right)\right. \\
& \left.+\bar{\theta}_{x x x} \phi-\bar{\theta} \phi_{x x x}-\bar{\theta}_{x x} \phi_{x}+\bar{\theta}_{x} \phi_{x x}\right], \\
\left(J_{0}^{(0)}\right)_{33}= & -i f \bar{\theta} \theta-i \int_{-\infty}^{x} f_{x} \bar{\theta} \theta d x^{\prime}+\varepsilon\left[4\left(\bar{\phi} \bar{\theta}_{x} \phi \phi-\phi_{x} \phi \bar{\phi} \bar{\theta}\right)+2\left(\bar{\phi}_{x} \bar{\theta} \phi \phi-\phi_{x} \phi \bar{\phi} \bar{\theta}\right.\right. \\
& \left.\left.-\bar{\theta} \phi \theta \bar{\theta}_{x}\right)+\bar{\theta}_{x x x} \theta-\bar{\theta} \theta_{x x x}-\bar{\theta}_{x x} \theta_{x}+\bar{\theta}_{x} \theta_{x x}\right] .
\end{aligned}
$$

Since $J_{0}=J_{0}^{(0)}+J_{0}^{(1)}$, it is easy to draw the following conclusion 


$$
J_{0}=\left(\begin{array}{lll}
\left(J_{0}^{(0)}\right)_{11} & \left(J_{0}^{(1)}\right)_{12} & \left(J_{0}^{(1)}\right)_{13} \\
\left(J_{0}^{(1)}\right)_{21} & \left(J_{0}^{(0)}\right)_{22} & \left(J_{0}^{(0)}\right)_{23} \\
\left(J_{0}^{(1)}\right)_{31} & \left(J_{0}^{(0)}\right)_{32} & \left(J_{0}^{(0)}\right)_{33}
\end{array}\right) .
$$

In terms of the gauge transformation $\widetilde{\varphi}=g \varphi$, we obtain

$$
\widetilde{\varphi}_{x}=\hat{F} \widetilde{\varphi}, \quad \widetilde{\varphi}_{t}=\hat{G} \widetilde{\varphi},
$$

where $\hat{F}$ and $\hat{G}$ can be written as

$$
\begin{aligned}
\hat{F}= & g F g^{-1}+g_{x} g^{-1}=-i \lambda \Sigma+J_{1}, \\
\hat{G}= & g G g^{-1}+g_{t} g^{-1} \\
= & g\left(-\lambda f\left[S_{x}, S\right]-\left(i \lambda h+i \lambda^{2} f\right) S\right) g^{-1} \\
& +\varepsilon g\left(-i \lambda^{5} S+\lambda^{4}\left[S, S_{x}\right]+i \lambda^{3}\left(S_{x x}-3 S_{x} S_{x}+6 S S_{x} S_{x}\right)+\lambda^{2}\left(-\left[S, S_{x x x}\right]\right.\right. \\
& \left.+\left[S_{x}, S_{x x}\right]+10 S_{x} S_{x} S_{x}-20 S S_{x} S_{x} S_{x}\right)+i \lambda\left(-S_{x x x x}+5 S_{x x x} S_{x}\right. \\
& +5 S_{x x} S_{x x}+5 S_{x} S_{x x x}-10 S S_{x x x} S_{x}-10 S S_{x x} S_{x x}-10 S S_{x} S_{x x x} \\
& \left.-15 S_{x x} S_{x} S_{x}-10 S_{x} S_{x x} S_{x}-5 S_{x} S_{x} S_{x x}+35 S_{x} S_{x} S_{x} S_{x}-70 S S_{x} S_{x} S_{x} S_{x}\right) g^{-1} \\
& +J_{0} .
\end{aligned}
$$

Substituting (18) and (26) into (28), we obtain

$$
\widehat{F}=i\left(\begin{array}{ccc}
0 & \phi & \theta \\
\bar{\phi} & -\lambda & 0 \\
\bar{\theta} & 0 & -\lambda
\end{array}\right), \quad \widehat{G}=\left(\begin{array}{lll}
\widehat{G}_{11} & \widehat{G}_{12} & \widehat{G}_{13} \\
\widehat{G}_{21} & \widehat{G}_{22} & \widehat{G}_{23} \\
\widehat{G}_{31} & \widehat{G}_{32} & \widehat{G}_{33}
\end{array}\right),
$$

where 


$$
\begin{aligned}
& \widehat{G}_{11}=i f(\phi \bar{\phi}+\theta \bar{\theta})+i \int_{-\infty}^{x} f_{x}(\phi \bar{\phi}+\theta \bar{\theta}) d x^{\prime}+\varepsilon\left[6 \left(\phi_{x} \phi \bar{\phi} \bar{\phi}-\bar{\phi}_{x} \bar{\phi} \phi \phi+\phi_{x} \theta \bar{\phi} \bar{\theta}+\phi \theta_{x} \bar{\phi} \bar{\theta}\right.\right. \\
& \left.-\theta \phi \bar{\phi}_{x} \bar{\theta}-\theta \phi \bar{\phi} \bar{\theta}_{x}\right)+\bar{\phi} \phi_{x x x}-\bar{\phi}_{x x x} \phi-\bar{\phi}_{x} \phi_{x x}+\bar{\phi}_{x x} \phi_{x}+\theta_{x x x} \bar{\theta}-\theta \bar{\theta}_{x x x}-\theta_{x x} \bar{\theta}_{x} \\
& +\theta_{x} \bar{\theta}_{x x}-i \lambda^{3}(\phi \bar{\phi}+\theta \bar{\theta})+\lambda^{2}\left(\phi \bar{\phi}_{x}+\theta \bar{\theta}_{x}-\phi_{x} \bar{\phi}-\theta_{x} \bar{\theta}\right)+i \lambda\left(\phi_{x x} \bar{\phi}+\theta_{x x} \bar{\theta}\right. \\
& \left.\left.+\phi \bar{\phi}_{x x}+\theta \bar{\theta}_{x x}-\phi_{x} \bar{\phi}_{x}-\theta_{x} \bar{\theta}_{x}\right)+3 i \lambda \phi \bar{\phi} \phi \bar{\phi}+6 i \lambda \phi \bar{\phi} \theta \bar{\theta}\right], \\
& \widehat{G}_{12}=i \lambda f \phi-(f \phi)_{x}+i h \phi+\varepsilon\left[6 i(\phi \bar{\phi} \phi \bar{\phi} \phi+2 \theta \bar{\theta} \phi \bar{\phi} \phi)+i \phi_{x x x x}+2 i\left[\left(\phi \bar{\phi}_{x}\right)_{x} \phi+\left(\phi_{x} \bar{\phi}\right)_{x} \phi\right.\right. \\
& \left.+3\left(\phi_{x} \phi\right)_{x} \bar{\phi}+2\left(\theta_{x} \phi\right)_{x} \bar{\theta}+\left(\theta \phi_{x}\right)_{x} \bar{\theta}+\theta\left(\bar{\theta} \phi_{x}\right)_{x}+\left(\theta \bar{\theta}_{x}\right)_{x} \phi\right]+i \lambda^{4} \phi+\lambda^{3} \phi_{x} \\
& \left.-i \lambda^{2} \phi_{x x}-2 i \lambda^{2}(\phi \bar{\phi} \phi+\theta \bar{\theta} \phi)-\lambda \phi_{x x x}-6 \lambda \phi_{x} \bar{\phi} \phi-3 \lambda \theta_{x} \bar{\theta} \phi-3 \lambda \theta \bar{\theta} \phi_{x}\right] \text {, } \\
& \widehat{G}_{13}=i \lambda f \theta-(f \theta)_{x}+i h \theta+\varepsilon\left[6 i \phi \bar{\phi} \phi \bar{\phi} \theta+i \theta_{x x x x}+2 i\left[2\left(\phi_{x} \theta\right)_{x} \bar{\phi}+\left(\phi \bar{\phi}_{x}\right)_{x} \theta+\left(\bar{\phi} \theta_{x}\right)_{x} \phi\right.\right. \\
& \left.\left.+\left(\phi \theta_{x}\right)_{x} \bar{\phi}\right]+i \lambda^{4} \theta+\lambda^{3} \theta_{x}-i \lambda^{2} \theta_{x x}-2 i \lambda^{2} \phi \bar{\phi} \theta-\lambda \theta_{x x x}-3 \lambda \phi_{x} \bar{\phi} \theta-3 \lambda \phi \bar{\phi} \theta_{x}\right], \\
& \widehat{G}_{21}=i \lambda f \bar{\phi}+(f \bar{\phi})_{x}+i h \bar{\phi}+\varepsilon\left[6 i(\bar{\phi} \phi \bar{\phi} \phi \bar{\phi}+2 \bar{\phi} \phi \bar{\phi} \theta \bar{\theta})+i \bar{\phi}_{x x x x}+2 i\left[\left(\phi_{x} \bar{\phi}\right)_{x} \bar{\phi}\right.\right. \\
& \left.+\left(\phi \bar{\phi}_{x}\right)_{x} \bar{\phi}+3\left(\bar{\phi}_{x} \bar{\phi}\right)_{x} \phi+2 \theta\left(\bar{\phi} \bar{\theta}_{x}\right)_{x}+\theta\left(\bar{\phi}_{x} \bar{\theta}\right)_{x}+\left(\bar{\phi}_{x} \theta\right)_{x} \bar{\theta}+\left(\theta_{x} \bar{\theta}\right)_{x} \bar{\phi}\right]+i \lambda^{4} \bar{\phi} \\
& \left.-\lambda^{3} \bar{\phi}_{x}-i \lambda^{2} \bar{\phi}_{x x}-2 i \lambda^{2}(\bar{\phi} \phi \bar{\phi}+\bar{\phi} \theta \bar{\theta})+\lambda \bar{\phi}_{x x x}+6 \lambda \bar{\phi}_{x} \phi \bar{\phi}+3 \lambda \bar{\phi}_{x} \theta \bar{\theta}+3 \lambda \bar{\phi} \theta \bar{\theta}_{x}\right] \text {, } \\
& \widehat{G}_{22}=-\left(i \lambda h+i \lambda^{2} f\right)-i f \bar{\phi} \phi-i \int_{-\infty}^{x} f_{x} \bar{\phi} \phi d x^{\prime}+\varepsilon\left[6\left(\bar{\phi}_{x} \bar{\phi} \phi \phi-\phi_{x} \phi \bar{\phi} \bar{\phi}\right)+4\left(\phi \theta \bar{\phi}_{x} \bar{\theta}\right.\right. \\
& \left.-\phi_{x} \theta \bar{\phi} \bar{\theta}\right)+2\left(\phi \theta \bar{\phi} \bar{\theta}_{x}-\theta_{x} \phi \bar{\phi} \bar{\theta}\right)+\bar{\phi}_{x x x} \phi-\bar{\phi} \phi_{x x x}-\bar{\phi}_{x x} \phi_{x}+\bar{\phi}_{x} \phi_{x x}-i \lambda^{5} \\
& +i \lambda^{3} \phi \bar{\phi}+\lambda^{2}\left(\bar{\phi} \phi_{x}-\bar{\phi}_{x} \phi\right)-i \lambda\left(\bar{\phi}_{x x} \phi+\bar{\phi} \phi_{x x}-\bar{\phi}_{x} \phi_{x}\right) \\
& +5 i \lambda(\bar{\phi} \phi \bar{\phi} \phi+\bar{\phi} \theta \bar{\theta} \phi)-8 i \lambda \bar{\phi} \phi \bar{\phi} \phi-8 i \lambda \bar{\phi} \theta \bar{\theta} \phi] \text {, } \\
& \widehat{G}_{23}=-i f \bar{\phi} \theta-i \int_{-\infty}^{x} f_{x} \bar{\phi} \theta d x^{\prime}+\varepsilon\left[4\left(\bar{\phi}_{x} \bar{\phi} \phi \theta-\phi \theta_{x} \bar{\phi} \bar{\phi}\right)+2\left(\bar{\phi}_{x} \bar{\phi} \phi \theta-\phi_{x} \theta \bar{\phi} \bar{\phi}+\theta_{x} \bar{\theta} \bar{\phi} \theta\right)\right. \\
& +\bar{\phi}_{x x x} \theta-\bar{\phi} \theta_{x x x}-\bar{\phi}_{x x} \theta_{x}+\bar{\phi}_{x} \theta_{x x}+i \lambda^{3} \bar{\phi} \theta+\lambda^{2}\left(\bar{\phi} \theta_{x}-\bar{\phi}_{x} \theta\right) \\
& \left.-i \lambda\left(\bar{\phi}_{x x} \theta+\bar{\phi} \theta_{x x}-\bar{\phi}_{x} \theta_{x}\right)-3 i \lambda \bar{\phi} \phi \bar{\phi} \theta\right] \text {, } \\
& \widehat{G}_{31}=i \lambda f \bar{\theta}+(f \bar{\theta})_{x}+i h \bar{\theta}+\varepsilon\left[6 i \bar{\phi} \phi \bar{\phi} \phi \bar{\theta}+i \bar{\theta}_{x x x x}+2 i\left[2\left(\bar{\phi}_{x} \bar{\theta}\right)_{x} \phi+\left(\bar{\phi} \phi_{x}\right)_{x} \bar{\theta}+\left(\phi \bar{\theta}_{x}\right)_{x} \bar{\phi}\right.\right. \\
& \left.\left.+\left(\bar{\phi} \bar{\theta}_{x}\right)_{x} \phi\right]+i \lambda^{4} \bar{\theta}-\lambda^{3} \bar{\theta}_{x}-i \lambda^{2} \bar{\theta}_{x x}-2 i \lambda^{2} \bar{\theta} \phi \bar{\phi}+\lambda \bar{\theta}_{x x x}+3 \lambda \bar{\theta}_{x} \phi \bar{\phi}+3 \lambda \bar{\theta} \phi \bar{\phi}_{x}\right], \\
& \widehat{G}_{32}=-i f \bar{\theta} \phi-i \int_{-\infty}^{x} f_{x} \bar{\theta} \phi d x^{\prime}+\varepsilon\left[4\left(\bar{\phi} \bar{\theta}_{x} \phi \phi-\phi_{x} \phi \bar{\phi} \bar{\theta}\right)+2\left(\bar{\phi}_{x} \bar{\theta} \phi \phi-\phi_{x} \phi \bar{\phi} \bar{\theta}-\bar{\theta} \phi \theta \bar{\theta}_{x}\right)\right. \\
& +\bar{\theta}_{x x x} \phi-\bar{\theta} \phi_{x x x}-\bar{\theta}_{x x} \phi_{x}+\bar{\theta}_{x} \phi_{x x}+i \lambda^{3} \bar{\theta} \phi+\lambda^{2}\left(\bar{\theta} \phi_{x}-\bar{\theta}_{x} \phi\right)-i \lambda\left(\bar{\theta} \phi_{x x}\right. \\
& \left.\left.+\bar{\theta}_{x x} \phi-\bar{\theta}_{x} \phi_{x}\right)-3 i \lambda \bar{\theta} \phi \bar{\phi} \phi\right] \text {, } \\
& \widehat{G}_{33}=-\left(i \lambda h+i \lambda^{2} f\right)-i f \bar{\theta} \theta-i \int_{-\infty}^{x} f_{x} \bar{\theta} \theta d x^{\prime}+\varepsilon\left[4\left(\bar{\phi} \bar{\theta}_{x} \phi \theta-\bar{\theta} \bar{\phi} \phi \theta_{x}\right)+2\left(\bar{\phi}_{x} \bar{\theta} \phi \theta\right.\right. \\
& \left.-\bar{\theta} \bar{\phi} \phi_{x} \theta\right)+\bar{\theta}_{x x x} \theta-\bar{\theta} \theta_{x x x}-\bar{\theta}_{x x} \theta_{x}+\bar{\theta}_{x} \theta_{x x}-i \lambda^{5}+i \lambda^{3} \bar{\theta} \theta \\
& \left.+\lambda^{2}\left(\bar{\theta} \theta_{x}-\bar{\theta}_{x} \theta\right)-i \lambda\left(\bar{\theta} \theta_{x x}+\bar{\theta}_{x x} \theta-\bar{\theta}_{x} \theta_{x}\right)-3 i \lambda \bar{\theta} \phi \bar{\phi} \theta\right] \text {. }
\end{aligned}
$$

By virtue of the zero-curvature formulation of $\hat{F}$ and $\hat{G}$, we derive the super fifthorder inhomogeneous NLSE with the constraint (i) 


$$
\begin{aligned}
i \phi_{t} & -i(h \phi)_{x}+f\left[\phi_{x x}+2(\phi \bar{\phi}+\theta \bar{\theta}) \phi\right]+2 f_{x} \phi_{x}+2 \phi \int_{-\infty}^{x} f_{x} \bar{\phi} \phi d x^{\prime} \\
& +\theta \int_{-\infty}^{x} f_{x} \bar{\theta} \phi d x^{\prime} \\
& +\phi \int_{-\infty}^{x} f_{x} \theta \bar{\theta} d x^{\prime}+\varepsilon\left[-6 i(\phi \bar{\phi} \phi \bar{\phi} \phi+2 \theta \bar{\theta} \phi \bar{\phi} \phi)_{x}-i \phi_{x x x x x}\right. \\
& -2 i\left[\left(\phi \bar{\phi}_{x}\right)_{x} \phi+\left(\phi_{x} \bar{\phi}\right)_{x} \phi\right. \\
& \left.+3\left(\phi_{x} \phi\right)_{x} \bar{\phi}+2\left(\theta_{x} \phi\right)_{x} \bar{\theta}+\left(\theta \phi_{x}\right)_{x} \bar{\theta}+\theta\left(\bar{\theta} \phi_{x}\right)_{x}+\left(\theta \bar{\theta}_{x}\right)_{x} \phi\right]_{x} \\
& +12 i\left(\phi \bar{\phi}_{x} \phi \bar{\phi} \phi-\phi_{x} \phi \bar{\phi} \bar{\phi} \phi\right) \\
& -16 i \phi_{x} \bar{\phi} \phi \theta \bar{\theta}+12 i\left(\phi \bar{\phi}_{x} \phi \theta \bar{\theta}+\phi \phi \bar{\phi} \theta \bar{\theta}_{x}\right)-8 i \phi \phi \bar{\phi} \theta_{x} \bar{\theta} \\
& +i\left(2 \phi \bar{\phi}_{x x x} \phi-2 \phi \bar{\phi} \phi_{x x x}\right. \\
& -2 \phi \bar{\phi}_{x x} \phi_{x}+2 \phi \bar{\phi}_{x} \phi_{x x}+2 \theta \bar{\theta}{ }_{x x x} \phi-\theta \bar{\theta} \phi_{x x x}-\theta \bar{\theta}_{x x} \phi_{x}+\theta \bar{\theta}_{x} \phi_{x x}-\theta_{x x x} \bar{\theta} \phi \\
& \left.\left.+\theta_{x x} \bar{\theta}_{x} \phi-\theta_{x} \bar{\theta}_{x x} \phi\right)\right]=0, \\
& i \theta_{t}-i(h \theta)_{x}+f\left(\theta_{x x}+2 \phi \bar{\phi} \theta\right)+2 f_{x} \theta_{x}+\phi \int_{-\infty}^{x} f_{x} \bar{\phi} \theta d x^{\prime}+\theta \int_{-\infty}^{x} f_{x} \phi \bar{\phi} d x^{\prime} \\
& +\varepsilon\left[-6 i(\phi \bar{\phi} \phi \bar{\phi} \theta)_{x}-i \theta_{x x x x}-2 i\left[2\left(\phi_{x} \theta\right)_{x} \bar{\phi}+\left(\phi \bar{\phi}_{x}\right)_{x} \theta+\left(\bar{\phi} \theta_{x}\right)_{x} \phi+\left(\phi \theta_{x}\right)_{x} \bar{\phi}\right]_{x}\right. \\
& +12 i \phi \bar{\phi} \phi \bar{\phi} \bar{\phi}_{x} \theta-8 i \phi_{x} \bar{\phi} \phi \bar{\phi} \theta-4 i \phi \bar{\phi} \phi \bar{\phi} \theta_{x}+i\left(2 \phi \bar{\phi}_{x x x} \theta-\phi \bar{\phi} \theta_{x x x}-\phi \bar{\phi} \bar{\phi}_{x x} \theta_{x}+\right. \\
& \left.\phi \bar{\phi}_{x} \theta_{x x}-2 \theta \bar{\theta}_{x x} \theta_{x}+2 \theta \bar{\theta}_{x} \theta \theta_{x x}-\phi_{x x x} \bar{\phi} \theta-\phi_{x} \bar{\phi}_{x x} \theta+\phi_{x x} \bar{\phi} \theta\right]=0,
\end{aligned}
$$

where $\phi(x, t)$ is bosonic filed and $\theta(x, t)$ is fermionic one.

If one sets $\varepsilon=0$, Eq. (31) reduces to the super Hirota equation [28]. If by setting $\varepsilon=0, h=0$, and $f=1$, Eq. (31) is reduced to super NLSE (4). Under the reduction $\varepsilon=1, h=0$, and $f=0$, Eq. (31) leads to the super fifth-order NLSE [30].

In the following part, we shall derive the Bäcklund transformation of Eq. (31). Taking the transformations $T=\frac{\widetilde{\varphi_{1}}}{\widetilde{\varphi_{2}}}$ and $\zeta=\frac{\widetilde{\varphi_{3}}}{\widetilde{\varphi_{2}}}$, Eq. (27) leads to the following equations:

$$
\begin{aligned}
T_{x} & =i \phi+i \theta \zeta-i \bar{\phi} T^{2}+i \lambda T, \\
\zeta_{x} & =i \bar{\theta} T-i \bar{\phi} T \zeta, \\
T_{t} & =\hat{G_{11}} T+\hat{G_{12}}+\hat{G_{13}} \zeta-\hat{G_{21}} T^{2}-\hat{G_{22}} T-\hat{G_{23}} T \zeta, \\
\zeta_{t} & =\hat{G_{31}} T+\hat{G_{32}}+\hat{G_{33}} \zeta-\hat{G_{21}} T \zeta-\hat{G_{22}} \zeta .
\end{aligned}
$$

where $T$ and $\zeta$ are the bosonic and fermionic functions, respectively. Next we assume that under the transformation

$$
T \rightarrow T, \zeta \rightarrow \zeta, \phi \rightarrow \phi^{\prime}, \theta \rightarrow \theta^{\prime}, \lambda \rightarrow \bar{\lambda},
$$

the forms Eq. (32) do not change, where $\bar{\lambda}$ is the conjugate of $\lambda$. Then we obtain

$$
\begin{aligned}
& T_{x}=i \phi^{\prime}+i \theta^{\prime} \zeta-i \bar{\phi}^{\prime} T^{2}+i \bar{\lambda} T, \\
& \zeta_{x}=i \bar{\theta}^{\prime} T-i \bar{\phi}^{\prime} T \zeta .
\end{aligned}
$$


Based on (32) and (34), we derive the Bäcklund transformation

$$
\begin{aligned}
\phi-\phi^{\prime} & =\frac{T(\bar{\lambda}-\lambda)}{1+|T|^{2}-\zeta \bar{\zeta}}, \\
\theta-\theta^{\prime} & =\frac{T \zeta(\bar{\lambda}-\lambda)}{1+|T|^{2}-\zeta \bar{\zeta}} .
\end{aligned}
$$

Since $\phi=0, \theta=0$ are the trivial solution of (31), based on the Bäcklund transformation (35), we obtain a new solution of (31)

$$
\begin{aligned}
\phi^{\prime} & =\frac{\left(\sqrt{-\frac{\rho}{t}}-\sqrt{-\frac{\bar{\rho}}{t}}\right) \mu \exp \left(i \sqrt{-\frac{\rho}{t}} x\right)}{1+|\mu|^{2}-\delta \bar{\delta}|\omega|^{2}}, \\
\theta^{\prime} & =\frac{\left(\sqrt{-\frac{\rho}{t}}-\sqrt{-\frac{\bar{\rho}}{t}}\right) \mu \exp \left(i \sqrt{-\frac{\rho}{t}} x\right) \bar{\delta} \bar{\omega}}{1+|\mu|^{2}-\delta \bar{\delta}|\omega|^{2}},
\end{aligned}
$$

where $\rho, \mu$ and $\omega$ are the bosonic constants and $\delta$ is fermionic one.

\subsection{Constraint (ii) $S^{2}=3 S-21$}

Now one turns to the second constraint $S^{2}=3 S-2 I$. One derives $S_{t}$ and $\left[S, S_{x x}\right]$ satisfying $S S_{t} S=2 S_{t}$ and $S\left[S, S_{x x}\right] S=2\left[S, S_{x x}\right]$. Thus the deformation term $D$ should satisfy the equation

$$
S D+D S=3 D .
$$

Following the similar procedure as before, we obtain the fifth-order inhomogeneous HS model under the constraint (ii)

$$
\begin{aligned}
i S_{t}= & f\left[S, S_{x x}\right]+f_{x}\left[S, S_{x}\right]+i h S_{x}+i \varepsilon\left[S_{x x x x x}-15\left(S_{x x x} S_{x}+S_{x x} S_{x x}+S_{x} S_{x x x}\right.\right. \\
& \left.-S_{x x} S_{x} S_{x}\right)_{x}+10\left(S S_{x x x} S_{x}+S S_{x x} S_{x x}+S S_{x} S_{x x x}+S_{x} S_{x x} S_{x}\right)_{x} \\
& \left.+5\left(S_{x} S_{x} S_{x x}\right)_{x}-105\left(S_{x} S_{x} S_{x} S_{x}\right)_{x}+70\left(S S_{x} S_{x} S_{x} S_{x}\right)_{x}\right]
\end{aligned}
$$

The corresponding $F$ and $G$ can be expressed as

$$
\begin{aligned}
F= & -i \lambda S, \\
G= & -\lambda f\left[S_{x}, S\right]-\left(i \lambda h+i \lambda^{2} f\right) S+\varepsilon\left[-i \lambda^{5} S+\lambda^{4}\left[S, S_{x}\right]\right. \\
& +i \lambda^{3}\left(S_{x x}-9 S_{x} S_{x}+6 S S_{x} S_{x}\right) \\
& +\lambda^{2}\left(-\left[S, S_{x x x}\right]+\left[S_{x}, S_{x x}\right]+30 S_{x} S_{x} S_{x}-20 S S_{x} S_{x} S_{x}\right) \\
& +i \lambda\left(-S_{x x x x}+15 S_{x x x} S_{x}\right. \\
& +15 S_{x x} S_{x x}+15 S_{x} S_{x x x}-10 S S_{x x x} S_{x}-10 S S_{x x} S_{x x} \\
& -10 S S_{x} S_{x x x}-15 S_{x x} S_{x} S_{x} \\
& \left.\left.-10 S_{x} S_{x x} S_{x}-5 S_{x} S_{x} S_{x x}+105 S_{x} S_{x} S_{x} S_{x}-70 S S_{x} S_{x} S_{x} S_{x}\right)\right],
\end{aligned}
$$


where $\lambda$ is the spectral parameter.

Let

$$
J_{1}=i\left(\begin{array}{ccc}
0 & 0 & \theta_{1} \\
0 & 0 & \theta_{2} \\
\bar{\theta}_{1} & \bar{\theta}_{2} & 0
\end{array}\right) \in \mathrm{L}^{(1)} \text { for } S \in S U(2 / 1) / S(U(2) \times U(1))
$$

here $\theta_{1}(x, t), \theta_{2}(x, t)$ are the fermionic variables.

Substituting (14) and (19) into (38), we find

$$
\begin{aligned}
& {\left[\Sigma, J_{0}\right]=-i f\left[\Sigma,\left[\left[\Sigma, J_{1}\right], J_{1}\right]+\left[\Sigma, J_{1 x}\right]\right]-i f_{x}\left[\Sigma,\left[\Sigma, J_{1}\right]\right]+h\left[\Sigma, J_{1}\right]} \\
& +\varepsilon\left[4\left[\left[\left[\left[\Sigma, J_{1 x}\right], J_{1}\right], J_{1}\right], J_{1}\right]+6\left[\left[\left[\Sigma, J_{1 x x}\right], J_{1}\right], J_{1}\right]\right. \\
& +\left[\left[\left[\left[\left[\Sigma, J_{1}\right], J_{1}\right], J_{1}\right], J_{1}\right], J_{1}\right] \\
& +3\left[\left[\left[\left[\Sigma, J_{1}\right], J_{1 x},\right], J_{1}\right], J_{1}\right]+8\left[\left[\left[\Sigma, J_{1 x}\right], J_{1 x}\right], J_{1}\right]+4\left[\left[\Sigma, J_{1 x x x}\right], J_{1}\right] \\
& +2\left[\left[\left[\left[\Sigma, J_{1}\right], J_{1}\right], J_{1 x}\right], J_{1}\right]+3\left[\left[\left[\Sigma, J_{1}\right], J_{1 x x}\right], J_{1}\right] \\
& +4\left[\left[\left[\Sigma, J_{1 x}\right], J_{1}\right], J_{1 x}\right] \\
& +6\left[\left[\Sigma, J_{1 x x}\right], J_{1 x}\right]+\left[\left[\left[\left[\Sigma, J_{1}\right], J_{1}\right], J_{1}\right], J_{1 x}\right]+3\left[\left[\left[\Sigma, J_{1}\right], J_{1 x}\right], J_{1 x}\right] \\
& +4\left[\left[\Sigma, J_{1 x}\right], J_{1 x x}\right]+\left[\Sigma, J_{1 x x x x}\right]+\left[\left[\left[\Sigma, J_{1}\right], J_{1}\right], J_{1 x x}\right] \\
& +\left[\left[\Sigma, J_{1}\right], J_{1 x x x}\right] \\
& -15\left(\left(\left[\Sigma, J_{1 x x}\right]+\left[\left[\left[\Sigma, J_{1}\right], J_{1}\right], J_{1}\right]+2\left[\left[\Sigma, J_{1 x}\right], J_{1}\right]\right.\right. \\
& \left.+\left[\left[\Sigma, J_{1}\right], J_{1 x}\right]\right)\left[\Sigma, J_{1}\right] \\
& +\left(\left[\left[\Sigma, J_{1}\right], J_{1}\right]+\left[\Sigma, J_{1 x}\right]\right)\left(\left[\left[\Sigma, J_{1}\right], J_{1}\right]+\left[\Sigma, J_{1 x}\right]\right) \\
& +\left[\Sigma, J_{1}\right]\left(\left[\Sigma, J_{1 x x}\right]\right. \\
& +\left[\left[\left[\Sigma, J_{1}\right], J_{1}\right], J_{1}\right]+2\left[\left[\Sigma, J_{1 x}\right], J_{1}\right] \\
& \left.+\left[\left[\Sigma, J_{1}\right], J_{1 x}\right]\right)-\left(\left[\left[\Sigma, J_{1}\right], J_{1}\right]+\left[\Sigma, J_{1 x}\right]\right) \\
& \left.\left[\Sigma, J_{1}\right]\left[\Sigma, J_{1}\right]\right)_{x}+10\left(\Sigma \left(\left[\Sigma, J_{1 x x}\right]+\left[\left[\left[\Sigma, J_{1}\right], J_{1}\right], J_{1}\right]+2\left[\left[\Sigma, J_{1 x}\right], J_{1}\right]\right.\right. \\
& \left.+\left[\left[\Sigma, J_{1}\right], J_{1 x}\right]\right)\left[\Sigma, J_{1}\right]+\Sigma\left(\left[\left[\Sigma, J_{1}\right], J_{1}\right]\right. \\
& \left.+\left[\Sigma, J_{1 x}\right]\right)\left(\left[\left[\Sigma, J_{1}\right], J_{1}\right]+\left[\Sigma, J_{1 x}\right]\right) \\
& +\Sigma\left[\Sigma, J_{1}\right]\left(\left[\Sigma, J_{1 x x}\right]+\left[\left[\left[\Sigma, J_{1}\right], J_{1}\right], J_{1}\right]\right. \\
& \left.+2\left[\left[\Sigma, J_{1 x}\right], J_{1}\right]+\left[\left[\Sigma, J_{1}\right], J_{1 x}\right]\right) \\
& \left.+\left[\Sigma, J_{1}\right]\left(\left[\left[\Sigma, J_{1}\right], J_{1}\right]+\left[\Sigma, J_{1 x}\right]\right)\left[\Sigma, J_{1}\right]\right)_{x} \\
& +5\left([ \Sigma , J _ { 1 } ] [ \Sigma , J _ { 1 } ] \left(\left[\left[\Sigma, J_{1}\right], J_{1}\right]\right.\right. \\
& \left.\left.+\left[\Sigma, J_{1 x}\right]\right)\right)_{x}-105\left(\left[\Sigma, J_{1}\right]\left[\Sigma, J_{1}\right]\left[\Sigma, J_{1}\right]\left[\Sigma, J_{1}\right]\right)_{x} \\
& +70\left(\Sigma\left[\Sigma, J_{1}\right]\left[\Sigma, J_{1}\right]\right. \\
& \left.\left.\left[\Sigma, J_{1}\right]\left[\Sigma, J_{1}\right]\right)_{x}\right] \text {, }
\end{aligned}
$$

where $\Sigma=\operatorname{diag}(1,1,2)$.

Repeating the process of constraint (i), naturally, we obtain 


$$
J_{0}^{(0)}=\left(\begin{array}{ccc}
\left(J_{0}^{(0)}\right)_{11} & \left(J_{0}^{(0)}\right)_{12} & 0 \\
\left(J_{0}^{(0)}\right)_{21} & \left(J_{0}^{(0)}\right)_{22} & 0 \\
0 & 0 & \left(J_{0}^{(0)}\right)_{33}
\end{array}\right), J_{0}^{(1)}=\left(\begin{array}{ccc}
0 & 0 & \left(J_{0}^{(1)}\right)_{13} \\
0 & 0 & \left(J_{0}^{(1)}\right)_{23} \\
\left(J_{0}^{(1)}\right)_{31} & \left(J_{0}^{(1)}\right)_{32} & 0
\end{array}\right)
$$

Combining the two matrix in (42), we obtain

$$
J_{0}=\left(\begin{array}{lll}
\left(J_{0}^{(0)}\right)_{11} & \left(J_{0}^{(0)}\right)_{12} & \left(J_{0}^{(1)}\right)_{13} \\
\left(J_{0}^{(0)}\right)_{21} & \left(J_{0}^{(0)}\right)_{22} & \left(J_{0}^{(1)}\right)_{23} \\
\left(J_{0}^{(1)}\right)_{31} & \left(J_{0}^{(1)}\right)_{32} & \left(J_{0}^{(0)}\right)_{33}
\end{array}\right)
$$

where

$$
\begin{aligned}
& \left(J_{0}^{(0)}\right)_{11}=\text { if } \theta_{1} \bar{\theta}_{1}+i \int_{-\infty}^{x} f_{x} \theta_{1} \bar{\theta}_{1} d x^{\prime}+\varepsilon\left[4\left(\theta_{1} \theta_{2} \bar{\theta}_{2} \bar{\theta}_{1 x}-\theta_{1 x} \theta_{2} \bar{\theta}_{2} \bar{\theta}_{1}\right)-2\left(\bar{\theta}_{2} \bar{\theta}_{1} \theta_{1} \theta_{2 x}\right.\right. \\
& \left.\left.-\theta_{1} \theta_{2} \bar{\theta}_{2 x} \bar{\theta}_{1}\right)+\theta_{1 x x x} \bar{\theta}_{1}-\theta_{1} \bar{\theta}_{1 x x x}-\theta_{1 x x} \bar{\theta}_{1 x}+\theta_{1 x} \bar{\theta}_{1 x x}\right] \text {, } \\
& \left(J_{0}^{(0)}\right)_{12}=\text { if } \theta_{1} \bar{\theta}_{2}+i \int_{-\infty}^{x} f_{x} \theta_{1} \bar{\theta}_{2} d x^{\prime}+\varepsilon\left[2\left(\theta_{1} \bar{\theta}_{2 x} \theta_{2} \bar{\theta}_{2}-\theta_{1} \bar{\theta}_{1} \theta_{1 x} \bar{\theta}_{2}\right)+\theta_{1 x x x} \bar{\theta}_{2}\right. \\
& \left.-\theta_{1} \bar{\theta}_{2 x x x}-\theta_{1 x x} \bar{\theta}_{2 x}+\theta_{1 x} \bar{\theta}_{2 x x}\right] \text {, } \\
& \left(J_{0}^{(1)}\right)_{13}=i h \theta_{1}-\left(f \theta_{1}\right)_{x}+\varepsilon\left[-4 i\left(\theta_{1 x} \theta_{2}\right)_{x} \bar{\theta}_{2}-2 i\left[\bar{\theta}_{2}\left(\theta_{1} \theta_{2 x}\right)_{x}+\theta_{1}\left(\theta_{2 x} \bar{\theta}_{2}\right)_{x}\right.\right. \\
& \left.\left.-\left(\theta_{1} \bar{\theta}_{2 x}\right)_{x} \theta_{2}\right]+i \theta_{1 x x x x}\right] \text {, } \\
& \left(J_{0}^{(0)}\right)_{21}=\text { if } \theta_{2} \bar{\theta}_{1}+i \int_{-\infty}^{x} f_{x} \theta_{2} \bar{\theta}_{1} d x^{\prime}+\varepsilon\left[2\left(\theta_{2} \bar{\theta}_{1 x} \theta_{1} \bar{\theta}_{1}-\theta_{2} \bar{\theta}_{2} \theta_{2 x} \bar{\theta}_{1}\right)+\theta_{2 x x x} \bar{\theta}_{1}\right. \\
& \left.-\theta_{2} \bar{\theta}_{1 x x x}-\theta_{2 x x} \bar{\theta}_{1 x}+\theta_{2 x} \bar{\theta}_{1 x x}\right] \text {, } \\
& \left(J_{0}^{(0)}\right)_{22}=\text { if } \theta_{2} \bar{\theta}_{2}+i \int_{-\infty}^{x} f_{x} \theta_{2} \bar{\theta}_{2} d x^{\prime}+\varepsilon\left[4\left(\theta_{2} \bar{\theta}_{1} \bar{\theta}_{2 x} \theta_{1}-\theta_{2 x} \theta_{1} \bar{\theta}_{1} \bar{\theta}_{2}\right)-2\left(\bar{\theta}_{1} \theta_{2} \theta_{1 x} \bar{\theta}_{2}\right.\right. \\
& \left.\left.-\theta_{2} \bar{\theta}_{1 x} \bar{\theta}_{2} \theta_{1}\right)+\theta_{2 x x x} \bar{\theta}_{2}-\theta_{2} \bar{\theta}_{2 x x x}-\theta_{2 x x} \bar{\theta}_{2 x}+\theta_{2 x} \bar{\theta}_{2 x x}\right], \\
& \left(J_{0}^{(1)}\right)_{23}=i h \theta_{2}-\left(f \theta_{2}\right)_{x}+\varepsilon\left[-4 i\left(\theta_{2 x} \theta_{1}\right)_{x} \bar{\theta}_{1}-2 i\left[\bar{\theta}_{1}\left(\theta_{2} \theta_{1 x}\right)_{x}-\theta_{2}\left(\bar{\theta}_{1} \theta_{1 x}\right)_{x}\right.\right. \\
& \left.\left.-\left(\theta_{2} \bar{\theta}_{1 x}\right)_{x} \theta_{1}\right]+i \theta_{2 x x x x}\right] \text {, } \\
& \left(J_{0}^{(1)}\right)_{31}=i h \bar{\theta}_{1}+\left(f \bar{\theta}_{1}\right)_{x}+\varepsilon\left[-4 i\left(\bar{\theta}_{2} \bar{\theta}_{1 x}\right)_{x} \theta_{2}+2 i\left[\bar{\theta}_{1}\left(\bar{\theta}_{2 x} \theta_{2}\right)_{x}+\bar{\theta}_{2}\left(\theta_{2 x} \bar{\theta}_{1}\right)_{x}\right.\right. \\
& \left.\left.-\left(\bar{\theta}_{2 x} \bar{\theta}_{1}\right)_{x} \theta_{2}\right]+i \bar{\theta}_{1 x x x x}\right] \text {, } \\
& \left(J_{0}^{(1)}\right)_{32}=i h \bar{\theta}_{2}+\left(f \bar{\theta}_{2}\right)_{x}+\varepsilon\left[-4 i\left(\bar{\theta}_{1} \bar{\theta}_{2 x}\right)_{x} \theta_{1}+2 i\left[\bar{\theta}_{2}\left(\bar{\theta}_{1 x} \theta_{1}\right)_{x}+\bar{\theta}_{1}\left(\theta_{1 x} \bar{\theta}_{2}\right)_{x}\right.\right. \\
& \left.\left.-\left(\bar{\theta}_{1 x} \bar{\theta}_{2}\right)_{x} \theta_{1}\right]+i \bar{\theta}_{2 x x x x}\right] \text {, } \\
& \left(J_{0}^{(0)}\right)_{33}=i f\left(\theta_{1} \bar{\theta}_{1}+\theta_{2} \bar{\theta}_{2}\right)+i \int_{-\infty}^{x} f_{x}\left(\theta_{1} \bar{\theta}_{1}+\theta_{2} \bar{\theta}_{2}\right) d x^{\prime}+\varepsilon\left[6 \left(\bar{\theta}_{1} \theta_{1 x} \theta_{2} \bar{\theta}_{2}\right.\right. \\
& +\bar{\theta}_{2} \theta_{2 x} \theta_{1} \bar{\theta}_{1} \\
& \left.-\bar{\theta}_{2} \bar{\theta}_{1 x} \theta_{2} \theta_{1}-\bar{\theta}_{1} \bar{\theta}_{2 x} \theta_{1} \theta_{2}\right)+\bar{\theta}_{1 x x x} \theta_{1}-\bar{\theta}_{1} \theta_{1 x x x}-\bar{\theta}_{1 x x} \theta_{1 x}+\bar{\theta}_{1 x} \theta_{1 x x} \\
& \left.+\bar{\theta}_{2 x x x} \theta_{2}-\bar{\theta}_{2} \theta_{2 x x x}-\bar{\theta}_{2 x x} \theta_{2 x}+\bar{\theta}_{2 x} \theta_{2 x x}\right] \text {. }
\end{aligned}
$$


Due to the gauge transformationIn $\widetilde{\varphi}=g \varphi$, we obtain

$$
\widetilde{\varphi}_{x}=\widetilde{F} \widetilde{\varphi}, \quad \widetilde{\varphi}_{t}=\widetilde{G} \widetilde{\varphi},
$$

where $\widetilde{F}$ and $\widetilde{G}$ can be written as

$$
\begin{aligned}
\tilde{F}= & g U g^{-1}+g_{x} g^{-1}=-i \lambda \Sigma+J_{1}, \\
\tilde{G}= & g V g^{-1}+g_{t} g^{-1} \\
= & g\left(-\lambda f\left[S_{x}, S\right]-\left(i \lambda h+i \lambda^{2} f\right) S\right) g^{-1} \\
& +\varepsilon g\left[-i \lambda^{5} S+\lambda^{4}\left[S, S_{x}\right]+i \lambda^{3}\left(S_{x x}-3 S_{x} S_{x}+6 S S_{x} S_{x}\right)\right. \\
& +\lambda^{2}\left(-\left[S, S_{x x x}\right]+\left[S_{x}, S_{x x}\right]\right. \\
& \left.+10 S_{x} S_{x} S_{x}-20 S S_{x} S_{x} S_{x}\right)+i \lambda\left(-S_{x x x x}+5 S_{x x x} S_{x}+5 S_{x x} S_{x x}+5 S_{x} S_{x x x}\right. \\
& -10 S S_{x x x} S_{x}-10 S S_{x x} S_{x x}-10 S S_{x} S_{x x x}-15 S_{x x} S_{x} S_{x}-10 S_{x} S_{x x} S_{x} \\
& \left.\left.-5 S_{x} S_{x} S_{x x}+35 S_{x} S_{x} S_{x} S_{x}-70 S S_{x} S_{x} S_{x} S_{x}\right)\right] g^{-1}+J_{0} .
\end{aligned}
$$

By means of (40) and (43), we rewrite (46) as follows

$$
\tilde{F}=i\left(\begin{array}{ccc}
-\lambda & 0 & \theta_{1} \\
0 & -\lambda & \theta_{2} \\
\bar{\theta}_{1} & \overline{\theta_{2}} & -2 \lambda
\end{array}\right), \quad \tilde{G}=\left(\begin{array}{lll}
\tilde{G}_{11} & \tilde{G}_{12} & \tilde{G}_{13} \\
\tilde{G}_{21} & \tilde{G}_{22} & \tilde{G}_{23} \\
\tilde{G}_{31} & \tilde{G}_{32} & \tilde{G}_{33}
\end{array}\right),
$$

where 


$$
\begin{aligned}
& \tilde{G}_{11}=-\left(i \lambda h+i \lambda^{2} f\right)+i f \theta_{1} \bar{\theta}_{1}+i \int_{-\infty}^{x} f_{x} \theta_{1} \bar{\theta}_{1} d x^{\prime}+\varepsilon\left[-i \lambda^{5}-i \lambda^{3} \theta_{1} \bar{\theta}_{1}\right. \\
& -\lambda^{2}\left(\theta_{1 x} \bar{\theta}_{1}-\theta_{1} \bar{\theta}_{1 x}\right) \\
& +i \lambda\left(\theta_{1} \bar{\theta}_{1 x x}+\theta_{1 x x} \bar{\theta}_{1}\right)-i \lambda \theta_{1 x} \bar{\theta}_{1 x}+3 i \lambda \theta_{1} \bar{\theta}_{2} \theta_{2} \bar{\theta}_{1}+4\left(\theta_{1} \theta_{2} \bar{\theta}_{2} \bar{\theta}_{1 x}\right. \\
& \left.\left.-\theta_{1 x} \theta_{2} \bar{\theta}_{2} \bar{\theta}_{1}\right)-2\left(\bar{\theta}_{2} \bar{\theta}_{1} \theta_{1} \theta_{2 x}-\theta_{1} \theta_{2} \bar{\theta}_{2 x} \bar{\theta}_{1}\right)+\left(\theta_{1 x x x} \bar{\theta}_{1}-\theta_{1} \bar{\theta}_{1 x x x}-\theta_{1 x x} \bar{\theta}_{1 x}+\theta_{1 x} \bar{\theta}_{1 x x}\right)\right] \text {, } \\
& \tilde{G}_{12}=i f \theta_{1} \bar{\theta}_{2}+i \int_{-\infty}^{x} f_{x} \theta_{1} \bar{\theta}_{2} d x^{\prime}+\varepsilon\left[-i \lambda^{3} \theta_{1} \bar{\theta}_{2}-\lambda^{2}\left(\theta_{1 x} \bar{\theta}_{2}-\theta_{1} \bar{\theta}_{2 x}\right)+i \lambda\left(\theta_{1 x x} \bar{\theta}_{2}+\theta_{1} \bar{\theta}_{2 x x}\right)\right. \\
& \left.-i \lambda \theta_{1 x} \bar{\theta}_{2 x}+2\left(\theta_{1} \bar{\theta}_{2 x} \theta_{2} \bar{\theta}_{2}-\theta_{1} \bar{\theta}_{1} \theta_{1 x} \bar{\theta}_{2}\right)+\left(\theta_{1 x x x} \bar{\theta}_{2}-\theta_{1} \bar{\theta}_{2 x x x}-\theta_{1 x x} \bar{\theta}_{2 x}+\theta_{1 x} \bar{\theta}_{2 x x}\right)\right], \\
& \tilde{G}_{13}=i \lambda f \theta_{1}+i h \theta_{1}-\left(f \theta_{1}\right)_{x}+\varepsilon\left[i \lambda^{4} \theta_{1}+\lambda^{3} \theta_{1 x}-i \lambda^{2} \theta_{1 x x}+2 i \lambda^{2} \theta_{1} \theta_{2} \bar{\theta}_{2}-3 \lambda\left(\theta_{1 x} \bar{\theta}_{2} \theta_{2}\right.\right. \\
& \left.+\theta_{1} \bar{\theta}_{2} \theta_{2 x}\right)-\lambda \theta_{1 x x x}-4 i\left(\theta_{1 x} \theta_{2}\right)_{x} \bar{\theta}_{2}-2 i\left[\bar{\theta}_{2}\left(\theta_{1} \theta_{2 x}\right)_{x}\right. \\
& \left.\left.+\theta_{1}\left(\theta_{2 x} \bar{\theta}_{2}\right)_{x}-\left(\theta_{1} \bar{\theta}_{2 x}\right)_{x} \theta_{2}\right]+i \theta_{1 x x x x}\right] \text {, } \\
& \tilde{G}_{21}=i f \theta_{2} \bar{\theta}_{1}+i \int_{-\infty}^{x} f_{x} \theta_{2} \bar{\theta}_{1} d x^{\prime}+\varepsilon\left[-i \lambda^{3} \theta_{2} \bar{\theta}_{1}-\lambda^{2}\left(\theta_{2 x} \bar{\theta}_{1}-\theta_{2} \bar{\theta}_{1 x}\right)+i \lambda\left(\theta_{2 x x} \bar{\theta}_{1}+\theta_{2} \bar{\theta}_{1 x x}\right.\right. \\
& \left.\left.-\theta_{2 x} \bar{\theta}_{1 x}\right)+2\left(\theta_{2} \bar{\theta}_{1 x} \theta_{1} \bar{\theta}_{1}-\theta_{2} \bar{\theta}_{2} \theta_{2 x} \bar{\theta}_{1}\right)+\left(\theta_{2 x x x} \bar{\theta}_{1}-\theta_{2} \bar{\theta}_{1 x x x}-\theta_{2 x x} \bar{\theta}_{1 x}+\theta_{2 x} \bar{\theta}_{1 x x}\right)\right], \\
& \tilde{G}_{22}=-\left(i \lambda h+i \lambda^{2} f\right)+i f \theta_{2} \bar{\theta}_{2}+i \int_{-\infty}^{x} f_{x} \theta_{2} \bar{\theta}_{2} d x^{\prime}+\varepsilon\left[-i \lambda^{5}-i \lambda^{3} \theta_{2} \bar{\theta}_{2}-\lambda^{2}\left(\theta_{2 x} \bar{\theta}_{2}-\theta_{2} \bar{\theta}_{2 x}\right)\right. \\
& +i \lambda\left(\theta_{2} \bar{\theta}_{2 x x}+\theta_{2 x x} \bar{\theta}_{2}-\theta_{2 x} \bar{\theta}_{2 x}\right)+3 i \lambda \theta_{2} \bar{\theta}_{1} \theta_{1} \bar{\theta}_{2}+4\left(\theta_{2} \bar{\theta}_{1} \bar{\theta}_{2 x} \theta_{1}-\theta_{2 x} \theta_{1} \bar{\theta}_{1} \bar{\theta}_{2}\right) \\
& \left.-2\left(\bar{\theta}_{1} \theta_{2} \theta_{1 x} \bar{\theta}_{2}-\theta_{2} \bar{\theta}_{1 x} \bar{\theta}_{2} \theta_{1}\right)+\left(\theta_{2 x x x} \bar{\theta}_{2}-\theta_{2} \bar{\theta}_{2 x x x}-\theta_{2 x x} \bar{\theta}_{2 x}+\theta_{2 x} \bar{\theta}_{2 x x}\right)\right] \text {, } \\
& \tilde{G}_{23}=i \lambda f \theta_{2}+i h \theta_{2}-\left(f \theta_{2}\right)_{x}+\varepsilon\left[i \lambda^{4} \theta_{2}+\lambda^{3} \theta_{2 x}-i \lambda^{2} \theta_{2 x x}-2 i \lambda^{2} \theta_{2} \bar{\theta}_{1} \theta_{1}\right. \\
& -3 \lambda\left(\theta_{2} \bar{\theta}_{1} \theta_{1 x}+\theta_{2 x} \bar{\theta}_{1} \theta_{1}\right)-\lambda \theta_{2 x x x}-4 i\left(\theta_{2 x} \theta_{1}\right)_{x} \bar{\theta}_{1}-2 i\left[\bar{\theta}_{1}\left(\theta_{2} \theta_{1 x}\right)_{x}\right. \\
& \left.\left.-\theta_{2}\left(\bar{\theta}_{1} \theta_{1 x}\right)_{x}-\left(\theta_{2} \bar{\theta}_{1 x}\right)_{x} \theta_{1}\right]+i \theta_{2 x x x x}\right], \\
& \tilde{G}_{31}=i \lambda f \bar{\theta}_{1}+i h \bar{\theta}_{1}+\left(f \bar{\theta}_{1}\right)_{x}+\varepsilon\left[i \lambda^{4} \bar{\theta}_{1}-\lambda^{3} \bar{\theta}_{1 x}-i \lambda^{2} \bar{\theta}_{1 x x}-2 i \lambda^{2} \bar{\theta}_{2} \theta_{2} \bar{\theta}_{1}\right. \\
& +3 \lambda\left(\bar{\theta}_{2 x} \theta_{2} \bar{\theta}_{1}+\bar{\theta}_{2} \theta_{2} \bar{\theta}_{1 x}\right)+\lambda \bar{\theta}_{1 x x x}-4 i\left(\bar{\theta}_{2} \bar{\theta}_{1 x}\right)_{x} \theta_{2}+2 i\left[\bar{\theta}_{1}\left(\bar{\theta}_{2 x} \theta_{2}\right)_{x}\right. \\
& \left.\left.+\bar{\theta}_{2}\left(\theta_{2 x} \bar{\theta}_{1}\right)_{x}-\left(\bar{\theta}_{2 x} \bar{\theta}_{1}\right)_{x} \theta_{2}\right]+i \bar{\theta}_{1 x x x x}\right], \\
& \tilde{G}_{32}=i \lambda f \bar{\theta}_{2}+i h \bar{\theta}_{2}+\left(f \bar{\theta}_{2}\right)_{x}+\varepsilon\left[i \lambda^{4} \bar{\theta}_{2}-\lambda^{3} \bar{\theta}_{2 x}-i \lambda^{2} \bar{\theta}_{2 x x}-2 i \lambda^{2} \bar{\theta}_{1} \theta_{1} \bar{\theta}_{2}\right. \\
& +3 \lambda\left(\bar{\theta}_{1 x} \theta_{1} \bar{\theta}_{2}+\bar{\theta}_{1} \theta_{1} \bar{\theta}_{2 x}\right)+\lambda \bar{\theta}_{2 x x x}-4 i\left(\bar{\theta}_{1} \bar{\theta}_{2 x}\right)_{x} \theta_{1}+2 i\left[\bar{\theta}_{2}\left(\bar{\theta}_{1 x} \theta_{1}\right)_{x}\right. \\
& \left.\left.+\bar{\theta}_{1}\left(\theta_{1 x} \bar{\theta}_{2}\right)_{x}-\left(\bar{\theta}_{1 x} \bar{\theta}_{2}\right)_{x} \theta_{1}\right]+i \bar{\theta}_{2 x x x x}\right] \text {, } \\
& \tilde{G}_{33}=-2\left(i \lambda h+i \lambda^{2} f\right)+i f\left(\theta_{1} \bar{\theta}_{1}+\theta_{2} \bar{\theta}_{2}\right)+i \int_{-\infty}^{x} f_{x}\left(\theta_{1} \bar{\theta}_{1}+\theta_{2} \bar{\theta}_{2}\right) d x^{\prime}+\varepsilon\left[-2 i \lambda^{5}\right. \\
& +i \lambda^{3}\left(\bar{\theta}_{1} \theta_{1}+\bar{\theta}_{2} \theta_{2}\right)-\lambda^{2}\left(\bar{\theta}_{1 x} \theta_{1}-\bar{\theta}_{1} \theta_{1 x}+\bar{\theta}_{2 x} \theta_{2}-\bar{\theta}_{2} \theta_{2 x}\right) \\
& -i \lambda\left(\bar{\theta}_{1 x x} \theta_{1}+\bar{\theta}_{2 x x} \theta_{2}\right. \\
& \left.+\bar{\theta}_{2} \theta_{2 x x}+\bar{\theta}_{1} \theta_{1 x x}-\bar{\theta}_{1 x} \theta_{1 x}-\bar{\theta}_{2 x} \theta_{2 x}\right)-6 i \lambda \bar{\theta}_{1} \theta_{1} \bar{\theta}_{2} \theta_{2}+6\left(\bar{\theta}_{1} \theta_{1 x} \theta_{2} \bar{\theta}_{2}\right. \\
& +\bar{\theta}_{2} \theta_{2 x} \theta_{1} \bar{\theta}_{1} \\
& \left.-\bar{\theta}_{2} \bar{\theta}_{1 x} \theta_{2} \theta_{1}-\bar{\theta}_{1} \bar{\theta}_{2 x} \theta_{1} \theta_{2}\right)+\left(\bar{\theta}_{1 x x x} \theta_{1}-\bar{\theta}_{1} \theta_{1 x x x}-\bar{\theta}_{1 x x} \theta_{1 x}+\bar{\theta}_{1 x} \theta_{1 x x}\right) \\
& \left.+\left(\bar{\theta}_{2 x x x} \theta_{2}-\bar{\theta}_{2} \theta_{2 x x x}-\bar{\theta}_{2 x x} \theta_{2 x}+\bar{\theta}_{2 x} \theta_{2 x x}\right)\right] \text {. }
\end{aligned}
$$

According to the zero-curvature formulation of $\tilde{F}$ and $\tilde{G}$, we have the fermionic fifth-order inhomogeneous NLSEs, 


$$
\begin{aligned}
& i \theta_{1 t}-i\left(h \theta_{1}\right)_{x}+\left(f \theta_{1}\right)_{x x}+2 f \theta_{1} \bar{\theta}_{2} \theta_{2}-\theta_{1} \int_{-\infty}^{x} f_{x} \theta_{2} \bar{\theta}_{2} d x^{\prime}+\theta_{2} \int_{-\infty}^{x} f_{x} \theta_{1} \bar{\theta}_{2} d x^{\prime} \\
& +\varepsilon\left[4 i\left(\theta_{1 x} \theta_{2}\right)_{x x} \bar{\theta}_{2}+4 i\left(\theta_{1 x} \theta_{2}\right)_{x} \bar{\theta}_{2 x}+2 i\left[\bar{\theta}_{2}\left(\theta_{1} \theta_{2 x}\right)_{x}+\theta_{1}\left(\theta_{2 x} \bar{\theta}_{2}\right)_{x}-\left(\theta_{1} \bar{\theta}_{2 x}\right)_{x} \theta_{2}\right]_{x}\right. \\
& -i \theta_{1 x x x x x}+i \theta_{1}\left[\left(\bar{\theta}_{2 x} \theta_{2}-\bar{\theta}_{2} \theta_{2 x}\right)_{x x}-2\left(\bar{\theta}_{2 x x} \theta_{2 x}-\bar{\theta}_{2 x} \theta_{2 x x}\right)\right]+i\left[\left(\theta_{1 x} \bar{\theta}_{2}-\theta_{1} \bar{\theta}_{2 x}\right)_{x x}\right. \\
& \left.\left.-2\left(\theta_{1 x x} \bar{\theta}_{2 x}-\theta_{1 x} \bar{\theta}_{2 x x}\right)\right] \theta_{2}\right]=0 \text {, } \\
& i \theta_{2 t}-i\left(h \theta_{2}\right)_{x}+\left(f \theta_{2}\right)_{x x}+2 f \theta_{2} \bar{\theta}_{1} \theta_{1}-\theta_{2} \int_{-\infty}^{x} f_{x} \theta_{1} \bar{\theta}_{1} d x^{\prime}+\theta_{1} \int_{-\infty}^{x} f_{x} \theta_{2} \bar{\theta}_{1} d x^{\prime} \\
& +\varepsilon\left[4 i\left(\theta_{2 x} \theta_{1}\right)_{x x} \bar{\theta}_{1}+4 i\left(\theta_{2 x} \theta_{1}\right)_{x} \bar{\theta}_{1 x}+2 i\left[\bar{\theta}_{1}\left(\theta_{2} \theta_{1 x}\right)_{x}-\theta_{2}\left(\bar{\theta}_{1} \theta_{1 x}\right)_{x}-\left(\theta_{2} \bar{\theta}_{1 x}\right)_{x} \theta_{1}\right]_{x}\right. \\
& -i \theta_{2 x x x x}+i \theta_{2}\left[\left(\bar{\theta}_{1 x} \theta_{1}-\bar{\theta}_{1} \theta_{1 x}\right)_{x x}-2\left(\bar{\theta}_{1 x x} \theta_{1 x}-\bar{\theta}_{1 x} \theta_{1 x x}\right)\right]-i\left[\left(\theta_{2 x} \bar{\theta}_{1}-\theta_{2} \bar{\theta}_{1 x}\right)_{x x}\right. \\
& \left.\left.-2\left(\theta_{2 x x} \bar{\theta}_{1 x}-\theta_{2 x} \bar{\theta}_{1 x x}\right)\right] \theta_{1}\right]=0 \text {. }
\end{aligned}
$$

where $\theta_{1}(x, t), \theta_{2}(x, t)$ are the fermionic variables.

Specialising by setting $\varepsilon=0$, Eq. (48) reduces to the fermionic NLSE [28]. While when $\varepsilon=0, h=0$, and $f=1$, Eq. (48) leads to the fermionic NLSE (5). If one sets $\varepsilon=1, h=0$, and $f=0$, Eq. (48) reduces to the fermionic fifth-order NLSE [30].

Let us consider the transformation $T=\frac{\widetilde{\varphi_{1}}}{\widetilde{\varphi_{2}}}$ and $\zeta=\frac{\widetilde{\varphi_{3}}}{\widetilde{\varphi_{2}}}$, Eq. (45) leads to the following equations:

$$
\begin{gathered}
T_{x}=i \theta_{1} \zeta-i \theta_{2} T \zeta, \\
\zeta_{x}=i \overline{\theta_{1}} T+i \overline{\theta_{2}}-i \lambda \zeta, \\
T_{t}=\widetilde{G_{11}} T+\widetilde{G_{12}}+\widetilde{G_{13}} \zeta-\widetilde{G_{21}} T^{2}-\widetilde{G_{22}} T-\widetilde{G_{23}} T \zeta, \\
\zeta_{t}=\widetilde{G_{31}} T+\widetilde{G_{32}}+\widetilde{G_{33}} \zeta-\widetilde{G_{21}} T \zeta-\widetilde{G_{22}} T .
\end{gathered}
$$

Then we obain the Bäcklund transformation of the Eq. (48) as follows

$$
\begin{aligned}
\theta_{1}-\theta_{1}^{\prime} & =\frac{T \zeta(\bar{\lambda}-\lambda)}{1+|T|^{2}} \\
\theta_{2}-\theta_{2}^{\prime} & =\frac{\zeta(\bar{\lambda}-\lambda)}{1+|T|^{2}}
\end{aligned}
$$

where $T$ and $\zeta$ are the bosonic and fermionic functions, respectively.

Meanwhile, we can obtain a new nontrivial solution of (48)

$$
\begin{aligned}
& \theta_{1}^{\prime}=\frac{\left(\sqrt{-\frac{\rho}{t}}-\sqrt{-\frac{\bar{\rho}}{t}}\right) \mu \delta \exp \left(-i \sqrt{-\frac{\rho}{t}} x\right)}{1+|\mu|^{2}}, \\
& \theta_{2}^{\prime}=\frac{\left(\sqrt{-\frac{\rho}{t}}-\sqrt{-\frac{\bar{\rho}}{t}}\right) \delta \exp \left(-i \sqrt{-\frac{\rho}{t}} x\right)}{1+|\mu|^{2}},
\end{aligned}
$$


where $\rho$ is the bosonic constant, $\mu$ and $\delta$ are the fermionic constants.

\section{Summary and Discussion}

We establish the fifth-order inhomogeneous HS models with two different constraints and their Lax pairs have been derived. The corresponding gauge equivalent counterparts are the super and fermionic fifth-order inhomogeneous NLSEs, respectively. It is well-known that HS model has close relation with the strong electron related Hubbard model which has widely application in condensed matter physics. Therefore, the applications of the high-order inhomogeneous HS models should be of interest. The solutions of the integrable systems including HF model [31, 32] may provide new insight into the solutions of the HS models. In addition, it's worth noting that the Eq. (31) with bosonic limit and $\varepsilon=0$ can reduce to the generalized Hirota equation with $\gamma=0$. How to construct the fifth-order super Hirota equation with any parameter $\gamma$ and the corresponding deformed HS model still worth further discussing.

Acknowledgements This work is partially supported by National Natural Science Foundation of China (Grant Nos. 11965014 and 12061051) and Natural Science Foundation of Qinghai Province (Grant No. 2021-ZJ-708). The authors are particularly indebted to the anonymous reviewer of this paper for various kind suggestions and comments. The authors gratefully acknowledge the support of Professors $\mathrm{Ke} \mathrm{Wu}$ and Weizhong Zhao (CNU, China).

\section{Compliance with Ethical Statement}

Conflict of interest We declare that we do not have any commercial or associative interest that represents a conflict of interest in connection with the work submitted. Rong Han, Haichao Sun, Nana Jiang and Zhaowen Yan, November 1, 2020.

Open Access This article is licensed under a Creative Commons Attribution 4.0 International License, which permits use, sharing, adaptation, distribution and reproduction in any medium or format, as long as you give appropriate credit to the original author(s) and the source, provide a link to the Creative Commons licence, and indicate if changes were made. The images or other third party material in this article are included in the article's Creative Commons licence, unless indicated otherwise in a credit line to the material. If material is not included in the article's Creative Commons licence and your intended use is not permitted by statutory regulation or exceeds the permitted use, you will need to obtain permission directly from the copyright holder. To view a copy of this licence, visit http://creativecommons.org/licen ses/by/4.0/.

\section{References}

1. Lakshmanan, M.: Continuum spin system as an exactly solvable dynamical system. Phys. Lett. A 61, 53-54 (1977)

2. Zakharov, V.E., Takhtadzhyan, L.A.: Equivalence of the nonlinear Schrödinger equation and the equation of a Heisenberg ferromagnet. Theor. Math. Phys. 38, 17-23 (1979)

3. Lakshmanan, M., Ganesan, S.: Equivalent forms of a generalized Hirota's equation with linear inhomogeneities. J. Phys. Soc. Jpn. 52, 4031-4033 (1983)

4. Lakshmanan, M., Ganesan, S.: Geometrical and gauge equivalence of the generalized Hirota, Heisenberg and Wkis equations with linear inhomogeneities. Phys. A 132, 117-142 (1985) 
5. Sinha, D., Ghosh, P.K.: Integrable coupled Liénard-type systems with balanced loss and gain. Ann. Phys. 400, 109-127 (2019)

6. Saravanan, M., Cardoso, W.B.: Parametrically driven localized magnetic excitations with spatial inhomogeneity. Commun. Nonlinear. Sci. Num. Simulat. 69, 176-186 (2019)

7. Mikhailov, A.V., Shabat, A.B.: Integrable deformations of the Heisenberg model. Phys. Lett. A 116, 191-194 (1986)

8. Porsezian, K., Tamizhmani, K.M., Lakshmanan, M.: Geometrical equivalence of a deformed Heisenberg spin equation and the generalized nonlinear Schrödinger equation. Phys. Lett. A 124, 159-160 (1987)

9. Lakshmanan, M., Porsezian, K., Daniel, M.: Effect of discreteness on the continuum limit of the Heisenberg spin chain. Phys. Lett. A 133, 483-488 (1988)

10. Porsezian, K.: Nonlinear Schrödinger family on moving space curves: Lax pairs, soliton solution and equivalent spin chain. Chaos Solid. Fract. 9, 1709-1722 (1998)

11. Guo, J.F., Wang, S.K., Wu, K., Yan, Z.W., Zhao, W.Z.: Integrable higher order deformations of Heisenberg supermagnetic model. J. Math. Phys. 50, 113-502 (2009)

12. Zhao, W.Z., Bai, Y.Q., Wu, K.: Generalized inhomogeneous Heisenberg ferromagnet model and generalized nonlinear Schrödinger equation. Phys. Lett. A 352, 64-68 (2006)

13. Myrzakulov, R., Mamyrbekova, G., Nugmanova, G., Lakshmanan, M.: Integrable $(2+1)$-dimensional spin models with self-consistent potentials. Symmetry 7, 1352-1375 (2015)

14. Liu, Y.K., Li, B.: Rogue waves in the $(2+1)$-dimensional nonlinear Schrödinger equation with a parity-time-symmetric potential. Chin. Phys. Lett. 34, 010202 (2017)

15. Myrzakul, A., Myrzakulov, R.: Integrable geometric flows of interacting curves/surfaces, multilayer spin systems and the vector nonlinear Schrödinger equation. Int. J. Geom. 14, 1750136 (2017)

16. Ma, W.X., Shen, S.F., Yu, S.M., Zhang, H.Q., Zhang, W.Y.: An integrable so(3, R)-counterpart of the Heisenberg soliton hierarchy. Rep. Math. Phys. 74, 283-299 (2014)

17. Qiao, Z.J.: A finite-dimensional integrable system and the involutive solutions of the higher-order Heisenberg spin chain equations. Phys. Lett. A 186, 97-102 (1994)

18. Qiao, Z.J., Strampp, W.: On different integrable systems sharing the same nondynamical $r$-matrix. J. Math. Phys. 39, 3271-3279 (1998)

19. Bruce, A.J., Duplij, S.: Double-graded supersymmetric quantum mechanics. J. Math. Phys. 61, 063503 (2020)

20. Argurio, R., Bertolini, M., Franco, S., García-Valdecasas, E., Tatitscheff, V.: The Octagon and the non-supersymmetric string landscape. Phys. Lett. B 815, 136-153 (2021)

21. Aizawa, N., Amakawa, K., Doi, S.: $N$-extension of double-graded supersymmetric and superconformal quantum mechanics. J. Phys. A 53, 065205 (2020)

22. Mathieu, P.: Supersymmetric extension of the Korteweg-de Vries equation. J. Math. Phys. 29, 2499-2506 (1988)

23. Babalic, C.N., Carstea, A.S.: Bilinear approach to Kuperschmidt super-KdV type equations. J. Phys. A 51, 204-225 (2018)

24. Martin, Y.I., Radul, A.O.: A supersymmetric extension of the Kadomtsev-Petviashvili hierarchy. Commun. Math. Phys. 98, 65-77 (1985)

25. Makhankov, V.G., Pashaev, O.K.: Continual classical Heisenberg models defined on graded and algebras. J. Math. Phys.33, 2923-2936 (1992)

26. Yan, Z.W., Zhang, M.N., Ren, D.Y., Zhang, H., Cui, J.F.: On a super generalized $x$-dependent Hirota equation. Z. Naturforsch. A 72, 811-815 (2017)

27. Yan, Z.W., Zhang, M.N., Cui, J.F.: Higher-order inhomogeneous generalized Heisenberg supermagnetic model. Chin. Phys. Lett. 35, 050201 (2018)

28. Yan, Z.W., Gegenhasi.: On a integrable deformations of Heisenberg supermagnetic model. J. Nonlinear. Math. Phy. 23, 335-342 (2016)

29. Ma, W.X., He, J.S., Qin, Z.Y.: A supertrace identity and its applications to superintegrable systems. J. Math. Phys. 49, 033511 (2008)

30. Jiang, N.N., Zhang, M.N., Guo, J.F., Yan, Z.W.: Fifth-order generalized Heisenberg supermagnetic models. Chaos Solid Fract. 133, 109644 (2020)

31. Date, E., Jimbo, M., Kashiwara, M., Miwa, T.: Landau-Lifshitz equation: solitons, quasi-periodic solutions and infinite-dimensional Lie algebras. J. Phys. A 16, 221-236 (1983)

32. Ma, W.X., Zhou, Y.: Lump solutions to nonlinear partial differential equations via Hirota bilinear forms. J. Differ. Equ. 264, 2633-2659 (2018) 